\title{
Acid Sensing Ion Channels in Dorsal Spinal Cord Neurons
}

\author{
Anne Baron, Nicolas Voilley, Michel Lazdunski, and Eric Lingueglia \\ Institut de Pharmacologie Moléculaire et Cellulaire, Centre National de la Recherche Scientifique, Université de Nice Sophia-Antipolis, Unité Mixte de \\ Recherche 6097, Sophia Antipolis, 06560 Valbonne, France
}

\begin{abstract}
Acid-sensing ion channels (ASICs) are broadly expressed in the CNS, including the spinal cord. However, very little is known about the properties of ASICs in spinal cord neurons compared with brain. We show here that ASIC1a and ASIC2a are the most abundant ASICs in mouse adult spinal cord and are coexpressed by most neurons throughout all the laminas. ASIC currents in cultured embryonic day 14 mouse dorsal spinal neurons mainly flow through homomeric ASIC1a (34\% of neurons) and heteromeric ASIC1a plus 2a channels at a ratio of $2: 1$ ( $83 \%$ of neurons). ASIC2b only has a minor contribution to these currents. The two channel subtypes show different active $\mathrm{pH}$ ranges and different inactivation and reactivation kinetics supporting complementary functional properties. One striking property of native dorsal spinal neuron currents and recombinant currents is the $\mathrm{pH}$ dependence of the reactivation process. A light sustained acidosis induces a threefold slow-down of the homomeric ASICla (from pH 7.4 to $\mathrm{pH} 7.3$ ) and heteromeric ASICla plus 2a (from pH 7.4 to $\mathrm{pH} 7.2$ ) current reactivation ( $T_{0.5}$ increasing from 5.77 to $16.84 \mathrm{~s}$ and from 0.98 to $3.2 \mathrm{~s}$, respectively), whereas a larger acidosis to $\mathrm{pH} 6.6$ induces a 32-fold slow-down of the ASICla plus $2 \mathrm{a}$ current reactivation ( $T_{0.5}$ values increasing to $\left.31.30 \mathrm{~s}\right)$. The $\mathrm{pH}$ dependence of ASIC channel reactivation is likely to modulate neuronal excitability associated with repetitive firing in response to extracellular $\mathrm{pH}$ oscillations, which can be induced, for example, by intense synaptic activity of central neurons.
\end{abstract}

Key words: ASIC; spinal cord; PcTx1; reactivation; pain; acidosis

\section{Introduction}

Acid-sensing ion channels (ASICs) are neuronal voltageinsensitive cation channels gated by extracellular protons (Waldmann et al., 1997). Seven ASIC isoforms coded by four genes were described in mammals: the three splice variants ASIC1a, ASIC1b, and ASIC1b2; the two splice variants ASIC2a and ASIC2b; ASIC3; and ASIC4 (Price et al., 1996; Waldmann et al., 1996; Garcia-Anoveros et al., 1997; Lingueglia et al., 1997; Chen et al., 1998; Akopian et al., 2000; Grunder et al., 2000). Most of these isoforms can associate into homomeric or heteromeric channels (Lingueglia et al., 1997; Benson et al., 2002).

In sensory neurons, ASICs are associated with pain sensing and mechanoperception (McCleskey and Gold, 1999; Jones et al., 2004; Lingueglia, 2007). ASICs are also widely expressed in the CNS (Krishtal, 2003; Wemmie et al., 2006; Lingueglia, 2007). ASICs trigger action potentials (APs) after extracellular acidification, as shown in cortical and hippocampal neurons (Varming, 1999; Baron et al., 2002a). Several ASIC subunits are present in brain, including ASIC1a, ASIC2a, and ASIC2b, with significant overlap of expression (Krishtal, 2003; Voilley, 2004; Wemmie et al., 2006). Disrupting the ASIC1 gene eliminates the $\mathrm{H}^{+}$-evoked

Received July 2, 2007; revised Dec. 18, 2007; accepted Dec. 18, 2007.

This work was supported by the Centre National de la Recherche Scientifique, the Association Française contre les Myopathies, the Agence Nationale de la Recherche (ANR-05-NEUR-031), the Association pour la Recherche sur le Cancer, and the Institut National du Cancer. We are very grateful to Pierre Escoubas for kindly providing recombinant PcTx1.

Correspondence should be addressed to Anne Baron, Institut de Pharmacologie Moléculaire et Cellulaire, Centre National de la Recherche Scientifique, Université de Nice Sophia-Antipolis, Unité Mixte de Recherche 6097, 660 route des Lucioles, Sophia Antipolis, 06560 Valbonne, France. E-mail: baron@ipmc.cnrs.fr.

D01:10.1523/JNEUROSCI.4975-07.2008

Copyright $\odot 2008$ Society for Neuroscience $\quad$ 0270-6474/08/281498-11\$15.00/0 currents in hippocampal neurons (Wemmie et al., 2002) and in the amygdala (Wemmie et al., 2003), and these mice have a deficit in hippocampus-dependent spatial learning and cerebellumdependent eye-blink conditioning, associated with an impaired long-term potentiation (LTP) at Schaffer collateral-CA1 synapses and reduced EPSPs during high-frequency stimulation (Wemmie et al., 2002). The ASIC2a subunit appears to be important for the modulation of ASIC1a properties in brain (Askwith et al., 2004), and ASIC1a and ASIC2a are also important for neuronal function in the visual system (Ettaiche et al., 2004, 2006).

ASIC1a, ASIC2a, and ASIC2b are also expressed in the spinal cord (Wu et al., 2004), and $\mathrm{H}^{+}$-evoked ASIC-like transient cation currents were recorded in mouse and rat spinal neurons (Gruol et al., 1980; Li et al., 1997). Dorsal spinal cord neurons are the first central neurons to integrate and modulate the noxious signal generated by peripheral nociceptors. Dorsal horn is a highly plastic area, both at the morphological and functional level (Furue et al., 2004; Yang et al., 2004; Sandkuhler, 2007). Peripheral inflammation was shown to increase ASIC channel expression in spinal cord neurons (Wu et al., 2004), and ASICla has been involved previously in central sensitization (Duan et al., 2007). However, available data on ASIC channels in spinal cord neurons are still limited.

We analyzed the expression and the localization of the different ASIC isoforms in the mouse spinal cord and explored the functional properties of ASIC currents expressed by dorsal spinal neurons. The relative contribution of each ASIC isoform was determined with specific pharmacological tools, such as the PcTx1 toxin, a selective inhibitor of homomeric ASICla channels (Escoubas et al., 2000), and zinc, which is known to enhance ASIC2a-containing channels (Baron et al., 2001), and compared 
Table 1. Functional and pharmacological properties of dorsal spinal currents activated by a pH drop from pH 7.4 to pH 5.0 compared with those of heterologously expressed channels in COS cells

\begin{tabular}{|c|c|c|c|c|c|c|c|}
\hline Cell type & Current & $\mathrm{pH}_{0.5}$ activation & $\mathrm{pH}_{0.5}$ inactivation & Tau (s) inactivation & $T_{0.5}(\mathrm{~s})$ reactivation & Inhibition by PcTx1 & Stimulation by $\mathrm{Zn}^{2+}$ \\
\hline \multirow[t]{3}{*}{ Neurons } & Type 1 current & 6.46 & 7.30 & $1.00 \pm 0.06$ & 3.59 & Yes & No \\
\hline & Type 2 current & 6.03 & 6.74 & $0.76 \pm 0.03$ & 0.98 & No & Yes \\
\hline & $\mathrm{ASIC}^{-1-}$ current & 6.51 & 7.30 & $0.97 \pm 0.04$ & 5.49 & Yes & No \\
\hline \multirow[t]{5}{*}{ cos cells } & ASIC1a & 6.37 & 7.30 & $1.12 \pm 0.08$ & 11.05 & Yes & No \\
\hline & $\mathrm{ASIC} 2 \mathrm{a}$ & $3.87^{a}$ & 6.17 & $5.52 \pm 0.30$ & 0.92 & No & Yes \\
\hline & ASIC1a plus 2a (2:1) & 6.06 & 6.46 & $0.79 \pm 0.06$ & 0.50 & No & Yes \\
\hline & ASIC1a plus 2a (1:1) & $5.5^{a}$ & & $2.01 \pm 0.28$ & & No & Yes \\
\hline & ASIC1a plus 2a (1:2) & $5.1^{b}$ & & $3.53 \pm 0.79$ & & No & Yes \\
\hline
\end{tabular}

${ }^{a}$ Baron et al., 2001.

${ }^{b}$ Baron et al., 2002

with properties of dorsal spinal neurons from $\mathrm{ASIC}^{-/-}$mice. We also describe for the first time the steep $\mathrm{pH}$ dependency of ASIC channels reactivation, a new striking property of these channels with important consequences on neuronal excitability in spinal cord.

\section{Materials and Methods}

Heterologous expression of ASICs in COS cells. COS cells, at a density of 20,000 cells per $35 \mathrm{~mm}$ diameter Petri dish, were transfected using the DEAE-Dextran method, with pCI-ratASIC2a and/or pCI-ratASIC1a mixed with pCI-IRES-CD8 (1:5 ratio). Several transfection ratios (1:1, $1: 2$, or $2: 1$ ) were used to record ASIC1a plus 2a heteromers. The actual ratios of subunits within the heteromers formed are not known, but the currents resulting from the different transfection ratios have different functional properties (Table 1), indicating that the stoichiometry of subunits within the channel is influenced by the transfection ratio. Cells were used for electrophysiological measurements 1-3 d after transfection. Successfully transfected cells were recognized by their ability to fix CD8 antibody-coated beads (Dynal, Oslo, Norway).

Culture of mouse dorsal spinal neurons. After decapitation of a pregnant C57BL/6 mouse killed by $\mathrm{CO}_{2}$ inhalation, embryonic day 14 (E14) embryos were dissected in sterilized ice-cold medium (HBSS plus $6 \mathrm{~g} / \mathrm{L}$ glucose plus $10 \mathrm{~mm}$ HEPES, pH 7.4). The dorsal half of spinal cords were collected after a dorsal incision of embryos and cut into pieces. Two enzymatic incubations with $0.1 \%$ Trypsin (Seromed, Strasbourg, France) and 217 U/ml DNase I (type IV; Sigma, St. Quentin Fallavier, France), were performed at $37^{\circ} \mathrm{C}$ during $10 \mathrm{~min}$ each, followed by mechanical trituration with sigmacote-coated fire-polished Pasteur pipettes of decreasing diameters to finally obtain dissociated neurons. Trituration was performed in basal culture medium: Neurobasal (Invitrogen, Cergy Pontoise, France) plus 1\% Glutamax (Invitrogen) plus $15 \mu \mathrm{g} / \mathrm{ml} \mathrm{Genta-}$ mycine (Sigma) supplemented with $10 \%$ fetal calf serum (ICN Biochemicals, Orsay, France), 10\% dialysed, and heated inactivated horse serum (Sigma) and $217 \mathrm{U} / \mathrm{ml}$ DNase I. After centrifugation, neurons were plated at a density of 150,000 cells in $35 \mathrm{~mm}$ poly-L-lysine (Sigma)coated tissue culture plates (Falcon, Meylan, France), in basal culture medium with 2\% B27 supplement (Invitrogen), $5 \mu \mathrm{M}$ Uridine, and $5 \mu \mathrm{M}$ 5-Fluoro-2' deoxyuridine (Sigma), and kept in $95 \%$ air $/ 5 \% \mathrm{CO}_{2}$ at $37^{\circ} \mathrm{C}$. Cells were used for electrophysiological recordings from 1 to 3 weeks after plating, the culture medium being renewed twice each week.

Patch-clamp recordings. Ion currents were recorded using the wholecell patch-clamp technique (Hamill et al., 1981). Data were sampled at 10 $\mathrm{kHz}$ and low-pass filtered at $3 \mathrm{kHz}$ using pClamp8 software (Molecular Devices, Union City, CA). The statistical significance of differences between sets of data were estimated by the single-sided Student's $t$ test $\left({ }^{\star} p<\right.$ $\left.0.05,{ }^{* *} p<0.01,{ }^{* *} p<0.005\right)$. For COS cells, the pipette solution contained the following (in mM): $140 \mathrm{KCl}, 5 \mathrm{NaCl}, 2 \mathrm{MgCl}_{2}, 5 \mathrm{EGTA}, 10$ HEPES, pH 7.35, and the bath solution contained the following (in $\mathrm{mm}$ ): $150 \mathrm{NaCl}, 5 \mathrm{KCl}, 2 \mathrm{MgCl}_{2}, 2 \mathrm{CaCl}_{2}, 10 \mathrm{HEPES}, \mathrm{pH}$ 7.45. For neurons, 2 $\mathrm{mm} \mathrm{K}_{2} \mathrm{ATP}$ was added to the pipette solution, and $10 \mathrm{~mm}$ glucose was added to the bath solution, along with $20 \mu \mathrm{M}$ CNQX, $10 \mu \mathrm{M}$ kynurenic acid, and $10 \mu \mathrm{M}$ bicuculline (all from Sigma) to inhibit glutamate- and GABA-induced currents. MES was used instead of HEPES to buffer bath solution $\mathrm{pH}$ ranging from 6 to 5 . Changes in extracellular $\mathrm{pH}$ were induced by shifting one of eight outlets of a microperfusion system in front of the cell during $10 \mathrm{~s}$, unless otherwise stated. Experiments were performed at room temperature $\left(20-24^{\circ} \mathrm{C}\right)$. Recombinant PcTx1 (Psalmopoeus Cambridgei toxin 1) (Escoubas et al., 2003) was used, and $0.1 \%$ bovine serum albumin was added in extracellular solutions to prevent its adsorption to tubing and containers (Escoubas et al., 2000). PcTx1 was applied before and between $\mathrm{pH}$ drops, and the steady-state inhibition was measured after 3 min.

Quantitative reverse transcription-PCR. RNA was extracted from E14 and adult mouse spinal cord and from primary culture of mouse spinal cord neurons. After reverse transcription (RT) (with cloned AMV FirstStrand cDNA Synthesis kit; Invitrogen), quantitative PCR was performed using qPCR MasterMix Plus for SYBR Assay No ROX (Eurogentec, Seraing, Belgium) on a LightCycler480 (Roche Products, Welwyn Garden City, UK) following the protocol of the supplier. Primers were as follows: 18S, GCCGCTAGAGGTGAAATTCT/CATTCTTGGCAAATGCTTTCG; ASIC1a, GGCCAACTTCCGTAGCTTCA/ATGCCCTGCTCTGTCGTAGAA; ASIC1b, TCACCTAGGGACTTGGTGGC/ CCCTTCCACAAAGACATGGC; ASIC2a, CGACTTGTACCACGCTGGG/ATGCGGGTCAGGAATCTGC; ASIC2b, TCCCTTGGCTTGCTGTTGTC/CGTGTGTGACGGGAAACTGA; ASIC3, CACCCAATGACTTGCACTGG/TAGGCAGCATGTTCAGCAGG. Results were analyzed and plotted as relative expression to ASIC1a isoform expression level following the program for relative quantification supplied with the LightCycler.

ASIC localization by in situ hybridization and immunohistochemistry on spinal slices. For in situ hybridization, $12 \mu \mathrm{m}$ sections of frozen lumbar spinal cord from adult C57BL/6 mice were fixed with $4 \%$ paraformaldehyde, permeabilized with $0.1 \%$ Triton X-100, prehybridized, and hybridized with terminal-transferase biotin-dUTP oligonucleotide antisense probes against ASICla (CATTCTTGGAGACTTGGCTAAAGCGGAAC), ASIC2a (GCAGTAGAGCATCATATCCTTCAGGTCAT), and control sense probe (CACAGATGGCTGATGAAAAGCAG). After overnight incubation at $42^{\circ} \mathrm{C}$ and stringent wash, detection was performed using GenPoint Tyramide Signal Amplification System for Biotinylated Probes (DakoCytomation, Carpinteria, CA) according to the protocol of the supplier. The sections were counterstained with cresyl violet. Each experiment was done on three animals at least. Control experiments were done with sense probes (supplemental Fig. 2, available at www. jneurosci.org as supplemental material). For combined fluorescent in situ hybridization and immunohistochemistry, the detection was performed as follows. After stringent wash, sections were blocked with PBS containing $0.1 \%$ Tween $20,0.1 \%$ BSA and $2 \%$ horse serum and incubated overnight at $4^{\circ} \mathrm{C}$ with specific antibodies against biotin (1:5000 sheep antibody to biotin; Acris Antibodies, Hiddenhausen, Germany) and primary antibodies against ASIC2a (1:100; Alomone Labs, Jerusalem, Israel). Detection was done using Alexa Fluor 594 anti-sheep IgG and Alexa Fluor 488 anti-rabbit IgG (1:1000; Invitrogen). The specificity of the anti-ASIC2a antibody was confirmed by immunocytolabeling of ASIC2a-transfected COS cells and by double labeling by immunohistochemistry and in situ hybridization on spinal slices (supplemental Fig. 3, available at www.jneurosci.org as supplemental material). 
Results

Identification of three different types of ASIC-like currents in mouse dorsal spinal cord neurons

To analyze the functional properties of mouse spinal cord ASIC currents, we performed patch-clamp experiments on E14 primary cultured dorsal spinal neurons. In response to a $\mathrm{pH}$ drop from 7.4 to 5.0 , every recorded neuron expressed an ASIC current with a density of $120 \pm 6 \mathrm{pA} / \mathrm{pF}$ $(n=130)$. The mean cell capacitance of the recorded neurons was $30.6 \pm 0.8 \mathrm{pF}(n=$ $128)$, and their mean resting potential was $-51.55 \pm 0.95 \mathrm{mV}(n=87)$. Capsaicin (10 $\mu \mathrm{M})$ was not able to activate any current $(n=6)$.

Recombinant spider toxin PcTx1, at a concentration of $20 \mathrm{~nm}$ that selectively and fully inhibits heterologously expressed homomeric ASIC1a current (Escoubas et al., 2000), was tested on ASIC currents activated by a drop to $\mathrm{pH} 5.0$ (Fig. $1 A$ ). The effect of $\mathrm{Zn}^{2+}(300 \mu \mathrm{M})$ that increases the amplitude of ASIC currents flowing through ASIC2a-containing channels (Baron et al., 2001) was also tested on $\mathrm{pH}$ 6.3-induced currents, a more appropriate experimental condition to see maximal $\mathrm{Zn}^{2+}$ effect (Fig. 1C). PcTx1 inhibits ASIC current to $56 \pm 5 \%(n=47)$ of the control current (Fig. 1A, All), whereas $\mathrm{Zn}^{2+}$ increases the current amplitude $\left(\mathrm{IZn}^{2+} /\right.$ Icontrol $=1.76 \pm 0.19 ; n=29)($ Fig. $1 C$, All).

However, three different subpopulations of neurons could be identified, behaving differently with respect to PcTx1 and $\mathrm{Zn}^{2+}$ effect. Type 1 neurons, repre-

senting $17.2 \%$ of neurons (20 of 116), were defined as expressing a current highly inhibited by PcTx1 (IPcTx1/Icontrol from 19 to $0 \%$ of the control current; mean value, $11 \pm 2 \% ; n=12$ ) (Fig. $1 A)$ and not increased by $\mathrm{Zn}^{2+}\left(\mathrm{IZn}^{2+} /\right.$ Icontrol $=0.76 \pm 0.07$; $n=8$ ) (Fig. 1C). Such $\mathrm{Zn}^{2+}$-insensitive, PcTxl-sensitive ASIC current is expected to flow mainly through homomeric ASIC1a channels (Fig. $1 B, D$, left currents). In type 2 neurons, representing $66.4 \%$ of neurons (77 of 116), the ASIC current was almost insensitive to PcTx1, (IPcTx1/Icontrol from 100 to $75 \%$; mean value, $\pm 2 \% ; n=19$ ) (Fig. $1 A$ ), whereas $\mathrm{Zn}^{2+}$ produced an important increase in current amplitude $\left(\mathrm{IZn}^{2+} / I\right.$ control, $2.51 \pm$ $0.12 ; n=14$ ) (Fig. 1C). Therefore, this $\mathrm{Zn}^{2+}$-sensitive and PcTx1-insensitive current mainly flows through channels containing ASIC2a subunits (Fig. $1 B, D$, middle currents). The type 3 subpopulation of neurons was constituted by the remaining $16.4 \%$ of neurons (19 of 116), where PcTxl produced an incomplete and variable inhibition of the total ASIC current (IPcTx1/ Icontrol from 74 to $20 \%$ of the control current; mean value, $47 \pm$ $4 \% ; n=16$ ) (Fig. $1 A$ ), and $\mathrm{Zn}^{2+}$ produced a highly variable but significant increase in current amplitude $\left(\mathrm{IZn}^{2+} /\right.$ Icontrol, $1.51 \pm$ $0.53 ; n=6$ ) (Fig. $1 C$ ). In these neurons, the ASIC current appears to flow through a mixture of PcTx1-sensitive homomeric ASIC1a channels and of $\mathrm{Zn}^{2+}$-sensitive ASIC2a-containing channels
B

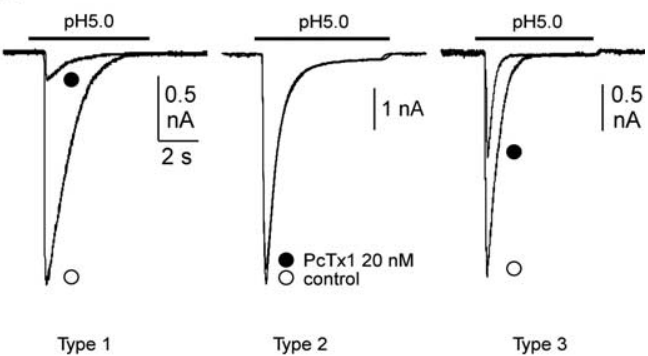

$\mathrm{D}$
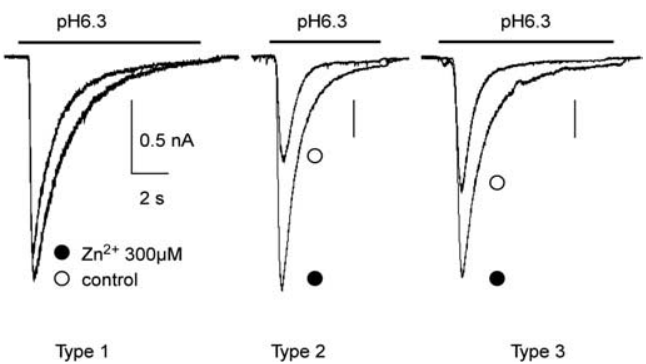

Type 3

rrded from mouse dorsalspinal neurons, $A$, Effect of $P$ cTx1. Currents Figure 1. Pharmacological properties of ASIC currents recorded from mouse dorsal spinal neurons. $A$, Effect of PCIX1. Currents
were recorded from wild-type neurons (All, type 1, type 2 , type 3 neurons) and from ASIC2 $2^{-1-}$ neurons, activated by pH drops 5.0 each minute, at a holding potential of $-50 \mathrm{mV}$.PcTx1 (20 nm) was applied between pH drops at pH 7.4. The current

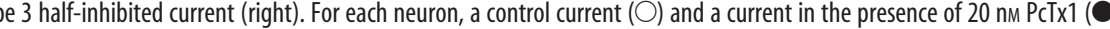
control) and plotted as mean \pm SEM ( $n$ ranging from 6 to $29 ;{ }^{*} p<0.05,{ }^{* * *} p<0.005$ compared with type 1 current; ${ }^{++} p<$ recorded from three different neurons showing the effect of zinc ( $300 \mu \mathrm{m})$ on a type 1 current (left), a type 2 current (middle), and a type 3 current (right). For each neuron, a control current $(\bigcirc)$ and a current in the presence of $300 \mu \mathrm{m}$ zinc $(\bigcirc)$ are shown. Currents were recorded at $-50 \mathrm{mV}$ and activated by a $\mathrm{pH}$ drop from 7.4 to 6.3 each minute.

(Fig. $1 B, D$, right currents). The ASIC current density, the cell capacitance, and the resting membrane potential were not significantly different between the three neuronal subpopulations, with current densities of $109 \pm 15,125 \pm 8$, and $104 \pm 13 \mathrm{pA} / \mathrm{pF}$; cell capacitances of $28.2 \pm 2.4,32 \pm 1$, and $27.7 \pm 2.5 \mathrm{pF}$; and resting potential of $-55.45 \pm 3.09,-51.54 \pm 1.1$, and $-50.40 \pm$ $2.81 \mathrm{mV}$ for type 1 , type 2 , and type 3 neurons, respectively.

Experiments were performed on ASIC currents recorded from dorsal spinal neurons from ASIC2 ${ }^{-1-}$ mice (Ettaiche et al., 2004). All ASIC2 ${ }^{-1-}$ neurons (cell capacitance, $28.0 \pm 1.6 \mathrm{pF}$; resting potential, $-50.13 \pm 1.86 \mathrm{mV} ; n=35)$ expressed an ASIC current $(86 \pm 10 \mathrm{pA} / \mathrm{pF} ; n=35)$ that was highly inhibited by PcTx1 (IPcTx1/Icontrol, $6 \pm 1 \%$ of the control current; $n=16$ ) (Fig. $1 \mathrm{~A})$ and that was not increased by $\mathrm{Zn}^{2+}\left(\mathrm{IZn}^{2+} /\right.$ Icontrol, $0.66 \pm 0.02 ; n=7$ ) (Fig. $1 C$ ), very similar to type 1 current.

In wild-type neurons, a sustained plateau phase following the type 2 or type 3 peak current was sometimes recorded (Fig. $1 B$ ), suggesting the participation of ASIC2b in some native heteromeric channels. Because ASIC2b confers a sustained plateau phase after, for instance, association with ASIC2a (Lingueglia et al., 1997), we performed experiments to analyze the possible implication of ASIC2b in type 2 current. In type 2 current of wildtype neurons, a sustained plateau phase of $2.70 \pm 0.28 \mathrm{pA} / \mathrm{pF}$ $(n=59)$ was recorded at $-50 \mathrm{mV}$. This current was reduced to 

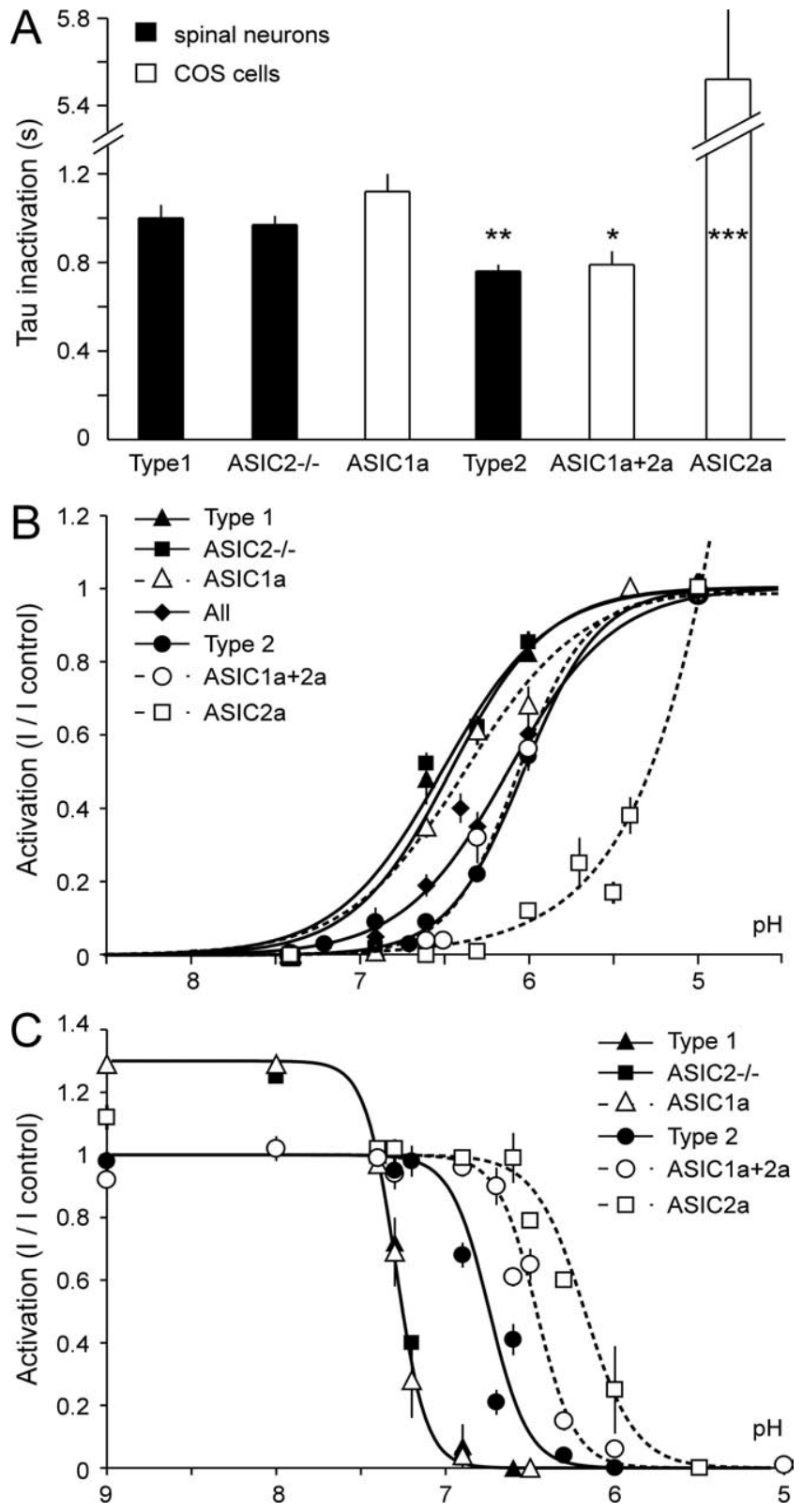

Figure 2. ASIC currents expressed by mouse dorsal spinal neurons mainly flow through ASIC1a and ASIC1a plus 2 a channels. $A$, Inactivation time course. Currents were recorded from neurons (black bars, type 1, type 2, and $\mathrm{ASIC}^{-1-}$ ) and from transfected COS cells (white bars, ASIC1a, ASIC2a, and ASIC1a plus 2a), activated by pH drops (10 s) from 7.4 to 5.0 , at a holding potential of $-50 \mathrm{mV}$. The inactivation time constant $\tau$ was measured by an exponential fit of the current decay $[I=$ $A^{*} \exp (-t / \tau)+C$, and plotted as mean \pm SEM ( $n$ ranging from 14 to $62 ;{ }^{*} p<0.05,{ }^{* *} p<0.01$, ${ }^{* * *} p<0.005$ compared with ASIC1a current). $\boldsymbol{B}$, pH-dependent activation. Currents were recorded from neurons (black symbols: $\boldsymbol{\Lambda}$, type 1; $\boldsymbol{\bullet}$, type 2; $\bullet$, All; $_{\mathbf{Q}}, \mathrm{ASIC}^{-1-}$ ) and from transfected COS cells (white symbols: $\triangle$, ASIC1a; $\square, A S I C 2 a ; \bigcirc, A S I C 1$ a plus $2 a$ ), activated by pHdrops (10s) from 7.4 to a variable $\mathrm{pH}$ test, at a holding potential of $-50 \mathrm{mV}$. The current amplitude was expressed as a ratio of the amplitude of the current elicited by a $\mathrm{pH}$ drop from $7.4 \mathrm{t} 05.0$ (I// control) and plotted as mean \pm SEM ( $n$ ranging from 3 to 73 ) as a function of the $\mathrm{pH}$ test. Data were fitted as a dose-response sigmoidal curve following the equation $Y=Y_{\min }+\left[\left(Y_{\max }-Y_{\min }\right) /\left(1+10^{\wedge}\left[\log \left(\mathrm{pH}_{0.5}-\right.\right.\right.\right.$ $\left.\left.X)^{*} n_{\mathrm{H}}\right]\right)$ ], where $\mathrm{pH}_{0.5}$ is $\mathrm{pH}$ of half-maximal activation, $n_{\mathrm{H}}$ is Hill number, $Y_{\text {min }}$ and $Y_{\text {max }}$ were 0 and 1 , respectively, except for ASIC2a $(\square)$, where $Y_{\text {max }}$ was 16 (incomplete curve shown). $\boldsymbol{\Delta}, \mathrm{pH}_{0.5}=6.46$, $n_{\mathrm{H}}=1.50 ; \boldsymbol{\square}, \mathrm{pH}_{0.5}=6.51, n_{\mathrm{H}}=1.39 ; \triangle, \mathrm{pH}_{0.5}=6.37, n_{\mathrm{H}}=1.25 ; \diamond, \mathrm{pH}_{0.5}=6.12, n_{\mathrm{H}}=1.39$; , $\mathrm{pH}_{0.5}=6.03, n_{\mathrm{H}}=1.94 ; \mathrm{O}, \mathrm{pH}_{0.5}=6.06, n_{\mathrm{H}}=2.15 ; \square, \mathrm{pH}_{0.5}=3.87, n_{\mathrm{H}}=1.05 . \mathrm{C}$, pH-dependent inactivation. Currents were recorded from neurons (black symbols: $\boldsymbol{\Lambda}$, type 1; $\mathbf{O}$, type 2; $\square$, ASIC2 $^{-1-}$ ) and from transfected COS cells (white symbols: $\triangle$, ASIC1a; $\square$, ASIC2a; $\bigcirc$, ASIC1a plus 2a), activated by pH drops (10 s) from a pH test to 5.0 , at a holding potential of $-50 \mathrm{mV}$. The current amplitude was expressed as a ratio of the amplitude of the current elicited by a $\mathrm{pH}$ drop from $\mathrm{pH} 7.4$ to 5.0 ( $/ / /$ control) and plotted as mean \pm SEM ( $n$ ranging from 4 to 24 ) as a function of the $\mathrm{pH}$
$1.24 \pm 0.15 \mathrm{pA} / \mathrm{pF}(n=50 ; p<0.005)$ in $\mathrm{ASIC}^{-/-}$neurons, strongly suggesting the involvement of ASIC2b subunits in the sustained plateau phase of wild-type type 2 current. The remaining plateau phase recorded in ASIC2 ${ }^{-1-}$ neurons was probably unrelated to the function of ASIC channels. Indeed, it was completely suppressed at $-70 \mathrm{mV}$, suggesting that it was generated by an $\mathrm{H}^{+}$-inhibited background $\mathrm{K}^{+}$current (data not shown) such as the two-pore domain potassium channel TASK (Duprat et al., 1997).

All these experiments suggest that the type 1 current is supported mainly by ASIC1a homomeric channels, whereas the type 2 current involves both ASIC1a and ASIC2a subunits, and the ASIC2b subunit when a plateau phase is expressed. Neurons displaying a type 3 current would express both ASIC1a subunits assembled into homomers, ASIC1a and ASIC2a subunits forming heteromers along with ASIC2b when there is a plateau phase.

\section{ASIC currents in dorsal spinal cord neurons flow through ASIC1a-containing channels with functional properties modulated by ASIC2a subunits}

Because the type 3 current is very variable and is in fact a mixture of different types of currents, the next series of experiments were particularly focused on a more extensive characterization of type 1 and type 2 currents. The inactivation time course, $\mathrm{pH}-$ dependent activation, $\mathrm{pH}$-dependent inactivation, and reactivation time course were compared between the type 1 current (mainly homomeric ASICla current), the type 2 current, the currents recorded from $\mathrm{ASIC}^{-1-}$ neurons (pure homomeric ASIC1a current), and heterologously expressed homomeric ASIC1a, homomeric ASIC2a, and heteromeric ASIC1a plus 2a currents in transfected COS cells (Table 1).

The inactivation time constant $\tau$ (Fig. $2 A$ ) was measured by an exponential fit of the $\mathrm{pH}$ 5.0-induced current decay $[I=$ $\left.A^{*} \exp (-t / \tau)+C\right]$. The $\tau$ values of the type 1 current $(1.00 \pm$ $0.06 \mathrm{~s} ; n=14)$ and of the ASIC2 ${ }^{-1-}$ current $(0.97 \pm 0.04 \mathrm{~s} ; n=$ 38) were not significantly different from the $\tau$ value of ASIC1a channels expressed in COS cells (1.12 $\pm 0.08 \mathrm{~s} ; n=34)$, as expected. Type 2 current showed a significant faster inactivation $\left.{ }^{* *} 0.76 \pm 0.03 \mathrm{~s} ; n=62\right)$ than the type 1 current, and also than the ASIC2a current expressed in COS cells ${ }^{* * *} \tau=5.52 \pm 0.30 \mathrm{~s}$; $n=23)$. The current recorded after cotransfection of ASIC1a and ASIC2a subunits in a $2: 1$ ratio showed a $\tau$ value $(0.79 \pm 0.06 \mathrm{~s}$; $n=13 ; p=0.74)$ similar to type 2 current. Comparatively, the current resulting from a 1:1 transfection ratio showed a $\tau=$ $2.01 \pm 0.28 \mathrm{~s}(n=6)$, and the current resulting from a 1:2 transfection ratio showed a $\tau=3.53 \pm 0.79 \mathrm{~s}(n=8)$ (data not shown).

Figure $2 \mathrm{~B}$ shows the $\mathrm{pH}$-dependent activation curves of ASIC currents. As expected, the type 1 current has activation properties similar to the ASIC2 ${ }^{-1-}$ current or the ASIC1 a current expressed in COS cells with $\mathrm{pH}_{0.5}$ values of 6.46, 6.51, and 6.37, respectively. The type 2 current was activated by more acidic $\mathrm{pH}\left(\mathrm{pH}_{0.5}=\right.$ 6.03). It is significantly different from the ASIC2a current expressed in COS cells $\left(\mathrm{pH}_{0.5}=3.87\right)$ (Baron et al., 2001). The current recorded after cotransfection of ASIC1a and ASIC2a subunits in a 2:1 ratio showed properties similar to the type 2 current in dorsal spinal neurons, with a $\mathrm{pH}_{0.5}=6.06$, whereas current

\section{$\leftarrow$}

test. Data were fitted as a dose-response sigmoidal curve following the equation $Y=Y_{\min }+$ $\left[\left(Y_{\max }-Y_{\min }\right) /\left(1+10^{\wedge}\left[\log \left(\mathrm{pH}_{0.5}-X\right)^{*} n_{\mathrm{H}}\right]\right)\right]$, where $\mathrm{pH}_{0.5}$ is $\mathrm{pH}$ of half-maximal activation, $n_{\mathrm{H}}$ is Hill number. $\mathbf{\Delta}, \boldsymbol{\square}, \triangle, \mathrm{pH}_{0.5}=7.30, n_{\mathrm{H}}=4.6, Y_{\max }=1.3 ; \boldsymbol{\bullet}, \mathrm{pH}_{0.5}=6.74, n_{\mathrm{H}}=3.82$; $\mathrm{O}, \mathrm{pH}_{0.5}=6.46 ; n_{\mathrm{H}}=3.82 ; \square, \mathrm{pH}_{0.5}=6.16, n_{\mathrm{H}}=3.82$. 
resulting from a 1:1 transfection ratio showed a $\mathrm{pH}_{0.5}$ of 5.5 (Baron et al., 2001; Chu et al., 2004; Hesselager et al., 2004), and the current resulting from a 1:2 transfection ratio showed a $\mathrm{pH}_{0.5}$ of 5.1 (Baron et al., 2002a).

The ASIC current types expressed in spinal dorsal neurons also have different $\mathrm{pH}$ dependences of inactivation (Fig. $2 \mathrm{C}$ ). The type 1 current and the ASIC2 ${ }^{-1-}$ current have the same $\mathrm{pH}_{0.5}$ of inactivation $\left(\mathrm{pH}_{0.5}=7.30\right)$ as the heterologously expressed homomeric ASICla current. The inactivation curve of the type 2 current is shifted toward more acidic $\mathrm{pH}$, with a $\mathrm{pH}_{0.5}=6.74$, intermediate between the $\mathrm{pH}_{0.5}$ values of heterologously expressed homomeric ASIC1a and ASIC2a currents that are 7.30 and 6.17, respectively. It is interesting to note that the ASIC1a current is already partly inactivated at $\mathrm{pH} 7.4$, contrary to the type 2 current. The current recorded after cotransfection of ASIC1a and ASIC2a subunits in a 2:1 ratio showed a $\mathrm{pH}_{0.5}$ of inactivation $\left(\mathrm{pH}_{0.5}=6.46\right)$, close to the $\mathrm{pH}_{0.5}$ value of the type 2 current.

Altogether, these functional properties support the fact that the type 1 current would mainly flow through homomeric ASIC1a channels, whereas the peak type 2 current would mainly flow through heteromeric ASIC1a plus 2a channels. The minor contribution of homomeric ASICla channels in type 2 current is responsible for the small PcTx1-induced inhibition ( $\sim 8 \%$ of the control current), but the contribution of homomeric ASIC2a can be ruled out, based on its atypical $\mathrm{pH}$-dependent activation and kinetics properties (see Table 1). The properties of type 2 current were close to those of heterologously expressed currents resulting from cotransfection of ASIC1a and ASIC2a in a 2:1 ratio, suggesting that it would mainly flow through ASIC1a plus $2 \mathrm{a}(2: 1)$ heteromers, although a minor participation of ASIC1a plus $2 \mathrm{a}(1: 2)$ cannot be ruled out. A major contribution of ASIC2b in association with ASIC1a can be excluded considering the functional properties of the ASIC1a plus 2b heteromers (Hesselager et al., 2004), which are not very different from those of ASICla alone and are not compatible with the functional properties of type 2 current. Type 3 neurons also expressed heteromeric ASIC1a plus 2a channels, mixed with a highly variable proportion of homomeric ASIC1a channels. These results clearly show that every cultured neuron expresses functional ASIC1a isoforms, either assembled into homomers in almost 34\% of the neurons (type 1 and type 3 neurons) or assembled into heteromers predominantly in association with ASIC2a in almost $83 \%$ of the neurons (type 2 and type 3 neurons).

\section{Reactivation of spinal current is $\mathrm{pH}$ dependent with important consequences on neuronal excitability}

After being inactivated by extracellular acidosis, ASIC currents reactivate after return to the resting $\mathrm{pH}$ value following a onephase exponential time course. To study this phenomenon on native spinal neuron currents, two successive $\mathrm{pH}$ drops separated by a variable time interval were applied to the same cell, and the amplitude of the second ASIC current was expressed as a ratio of the first control current amplitude and plotted as a function of the time interval duration (Fig. 3). The type 1 and the ASIC2 ${ }^{-1-}$ currents were very slow to reactivate $\left(T_{0.5}=3.59 \mathrm{~s}\right.$ and $T_{0.5}=$ $5.49 \mathrm{~s}$, respectively) compared with the type 2 current $\left(T_{0.5}=\right.$ $0.98 \mathrm{~s}$ ), consistent with data on recombinant ASIC1a and ASIC1a plus 2a channels (supplemental Fig. 1, available at www. jneurosci.org as supplemental material) (Benson et al., 2002). Reactivation kinetics thus constitute a major functional difference between the two subtypes of ASIC currents in dorsal spinal cord. Type 3 current reactivation kinetics were highly variable (data not shown), depending on the proportion of homomeric
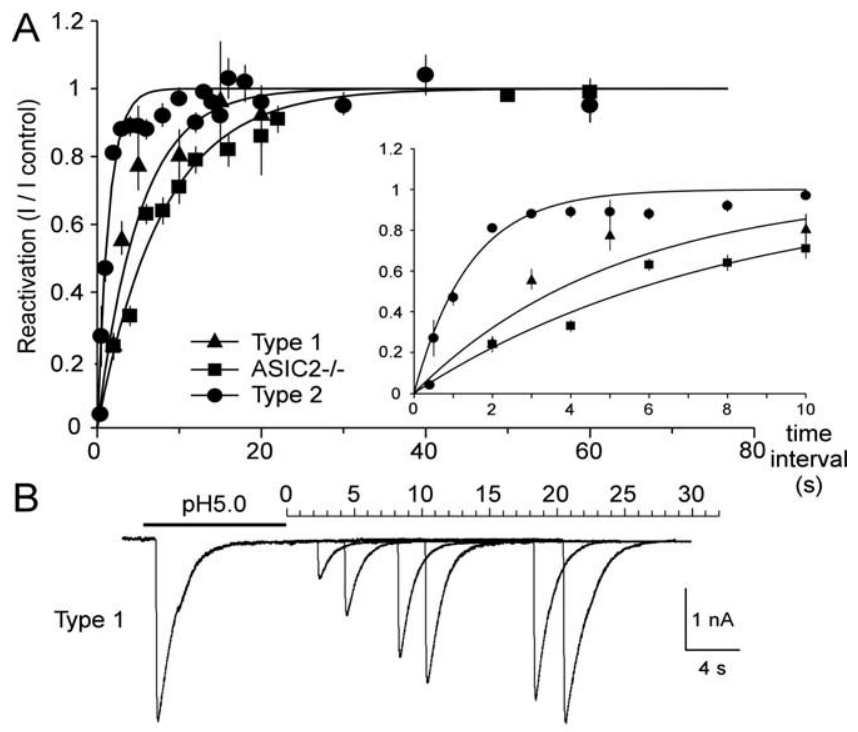

(s)
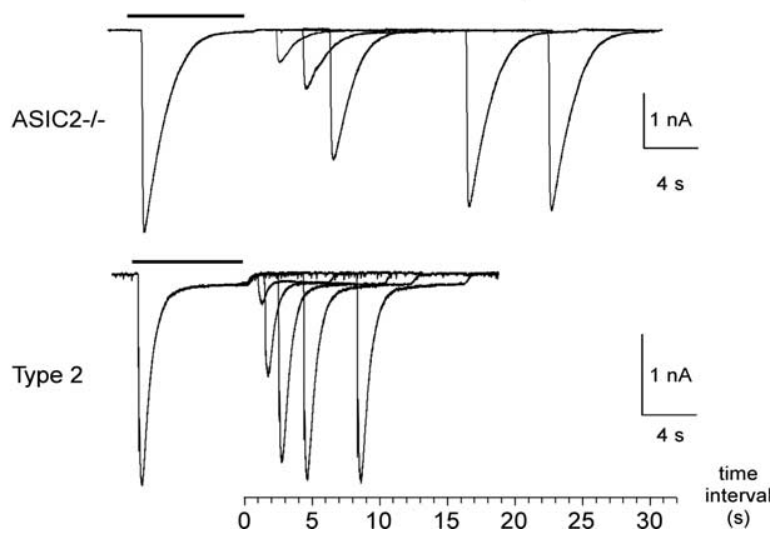

Figure 3. Reactivation time course of ASIC currents expressed by mouse dorsal spinal neurons. $\boldsymbol{A}$, Currents ( $\mathbf{\Delta}$, type $1 ; \boldsymbol{O}$, type $2 ; \mathbf{\square}, \mathrm{ASIC}^{-1-}$ ) were activated by repetitive $\mathrm{pH}$ drops from $\mathrm{pH} 7.4$ to 5.0 at a holding potential of $-50 \mathrm{mV}$. The current amplitude was expressed as a ratio of the amplitude of the control current ( $/ / /$ control) and plotted as mean \pm SEM ( $n$ ranging from 3 to 72 ) as a function of the time interval (s) between the end of the control $\mathrm{pH}$ drop and the onset of the next $\mathrm{pH}$ drop. Data were fitted as a one-phase exponential following the equation $Y=Y \max ^{*}\left[1-\exp \left(-k^{*} t\right)\right]$, with half reactivation time $T_{0.5}=0.69 / k . \mathbf{\Delta}, T_{0.5}=3.59 \mathrm{~s}$; $T_{0.5}=5.49 \mathrm{~s} ; \boldsymbol{O}, T_{0.5}=0.98 \mathrm{~s}$. Inset, Enlargement on the first $10 \mathrm{~s} . \boldsymbol{B}$, Original current traces recorded from three dorsal spinal neurons illustrating the difference of reactivation time courses between a type 1 current (top), an ASIC2 $2^{-1-}$ current (middle), and a type 2 current (bottom). For each neuron, several traces are superimposed, showing one control current and several subsequent reactivated currents recorded after different time intervals (s) from the end of the control pH drop: 2, 4, 8, 10, 18, and 20 s for type 1 current; $2,4,6,16$, and 22 s for ASIC2 ${ }^{-1-}$ current; and $0.5,1,2,4$, and $8 \mathrm{~s}$ for type 2 current. Currents were recorded at $-50 \mathrm{mV}$ and activated by repetitive $\mathrm{pH}$ drops from $\mathrm{pH} 7.4$ to 5.0 . Only the first $\mathrm{pH}$ drop corresponding to the control current is figured by a black line.

ASIC1a and heteromeric ASIC1a plus 2a channels involved in this mixed current.

In addition, we found the reactivation time course of ASIC currents of dorsal spinal neurons to be modulated by extracellular $\mathrm{pH}$ variations. The decrease of resting $\mathrm{pH}$ induced a major slow-down of reactivation of the type 2 current (Fig. $4 A$ ), with a 32-fold increase in $T_{0.5}$ value when resting $\mathrm{pH}$ decreases from 7.4 $\left(T_{0.5}=0.98 \mathrm{~s}\right)$ to $6.6\left(T_{0.5}=31.30 \mathrm{~s}\right)$. Representative current traces are shown in Figure $4 B$. This is a highly sensitive phenomenon. A very light acidosis from $\mathrm{pH} 7.4$ to $\mathrm{pH} 7.2$ is sufficient to increase $T_{0.5}$ from 0.98 up to $3.2 \mathrm{~s}$. Conversely, when the extracellular $\mathrm{pH}$ is increased from $\mathrm{pH} 7.4$ to $\mathrm{pH} 9.0$, it accelerates reactivation ( $T_{0.5}$ decreased from $0.98 \mathrm{~s}$ to $0.44 \mathrm{~s}$ ). This steep 

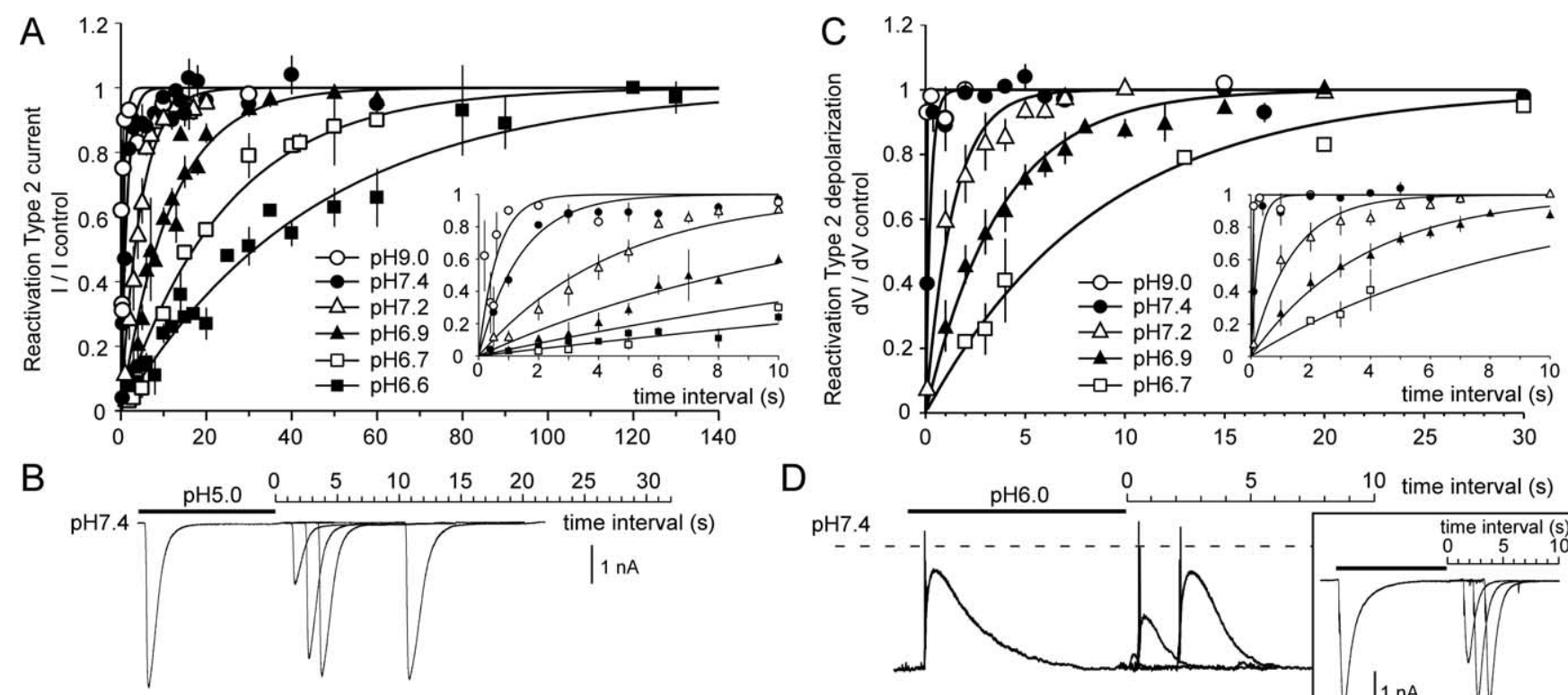

$\mathrm{D}$
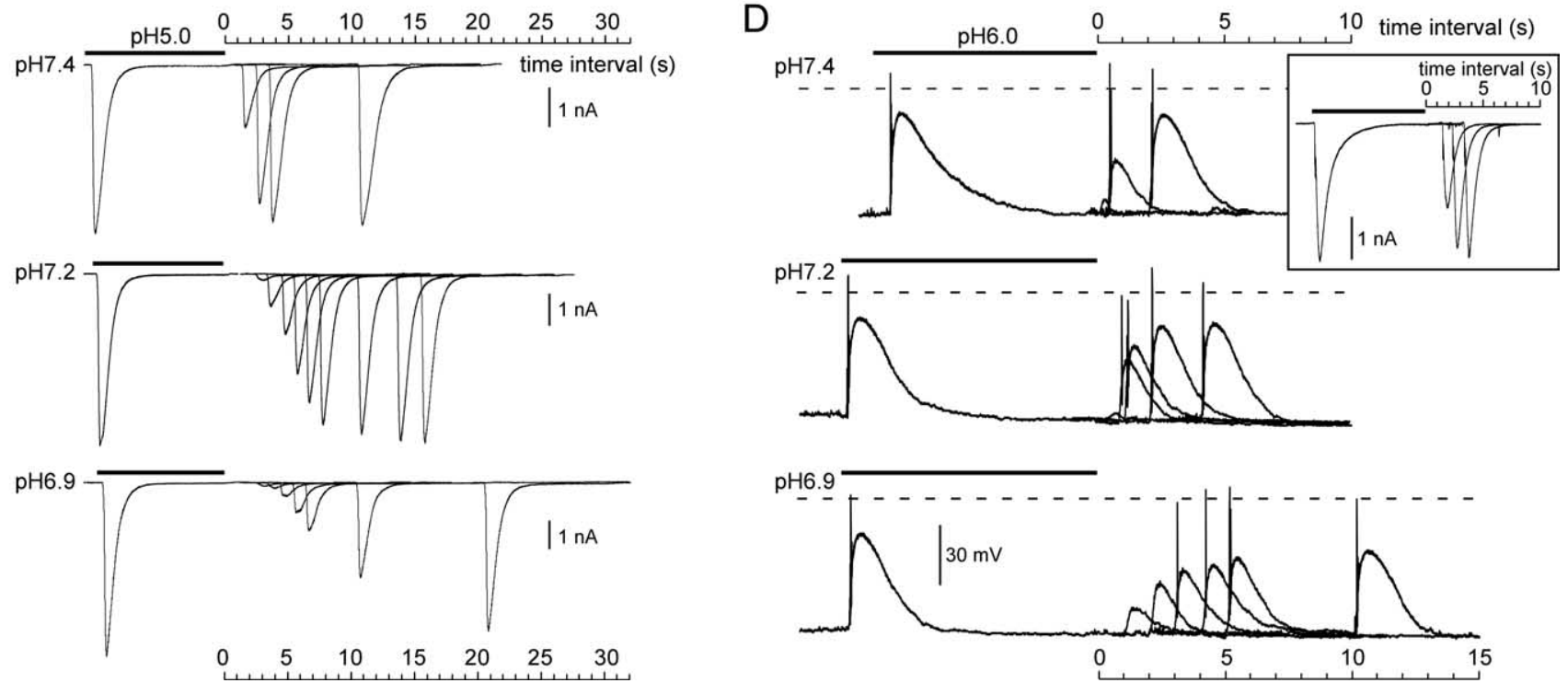

Figure 4. Modulation of the reactivation time course of dorsal spinal neuron type 2 current by extracellular $\mathrm{pH}$, and consequences on AP triggering. $A$, pH-dependent reactivation of type 2 current. Currents were recorded at a holding potential of $-50 \mathrm{mV}$ and activated by $\mathrm{pH}$ drops from various resting $\mathrm{pH}$ values to $\mathrm{pH}$ 5.0. The current amplitude was expressed as a ratio of the amplitude of the control current ( $/ / /$ control) and plotted as mean \pm SEM ( $n$ ranging from 3 to 72 ) as a function of the time interval (s) between the end of the control pH drop and the onset of the next pH drop. Data were fitted as a one-phase exponential following the equation: $Y=Y_{\max }^{*}\left[1-\exp \left(-k^{*} t\right)\right]$, with half-reactivation time $T_{0.5}=0.69 / k . \bigcirc, \mathrm{pH} 9.0, T_{0.5}=0.44 \mathrm{~s} ; \boldsymbol{\bullet}, \mathrm{pH} 7.4, T_{0.5}=0.98 \mathrm{~s} ; \triangle, \mathrm{pH}$ $7.2, T_{0.5}=3.21 \mathrm{~s} ; \boldsymbol{\Lambda}, \mathrm{pH} 6.9, T_{0.5}=8.13 \mathrm{~s} ; \square, \mathrm{pH} \mathrm{6.7,} T_{0.5}=17.03 \mathrm{~s} ; \mathbf{\square}, \mathrm{pH} 6.6, T_{0.5}=31.27 \mathrm{~s}$. Inset, Enlargement on the first $10 \mathrm{~s}$. $\boldsymbol{B}$, Original current traces recorded from one single neuron. Currents were recorded at $-50 \mathrm{mV}$ and activated by pH drops to pH 5.0 from pH 7.4 (top), pH 7.2 (middle), and pH 6.9 (bottom). For each resting pH value, several traces are superimposed, showing one control current and several subsequent reactivated currents recorded after different time intervals (s) from the end of the control pH drop: 1,2,3, and 10 sfor pH 7.4; 2, 3, 4, 5, 6, 7, 10, 13, and $15 \mathrm{~s}$ for $\mathrm{pH} 7.2$; and 2, 3, 4, 5, 6, 10, and $20 \mathrm{~s}$ for $\mathrm{pH} 6.9$. Only the first $\mathrm{pH}$ drop corresponding to the control current is figured by a black line. $\mathrm{C}$, pH-dependent reactivation of type 2 current-induced depolarization. Depolarizations were recorded in current-clamp mode from a mean membrane potential of $-51.54 \pm 1.1 \mathrm{mV}$ and activated by $\mathrm{pH}$ drops from various resting $\mathrm{pH}$ values to $\mathrm{pH} 5.0$ or $\mathrm{pH}$ 6.0. The depolarization amplitude was expressed as a ratio of the control depolarization ( $\mathrm{VV} / \mathrm{dV}$ control) and plotted as mean \pm SEM ( $n$ ranging from 3 to 18 ) as a function of the time interval (s) between the end of the control $\mathrm{pH}$ drop and the onset of the next $\mathrm{pH}$ drop. Data were fitted as a one-phase exponential following the equation: $Y=Y \max *\left[1-\exp \left(-k^{*} t\right)\right]$, with half reactivation time $T_{0.5}=0.69 / \mathrm{k} . \bigcirc, \mathrm{pH} \mathrm{9.0,} T_{0.5}=0.03 \mathrm{~s} ; \boldsymbol{O}, \mathrm{pH} 7.4, T_{0.5}=0.17 \mathrm{~s} ; \triangle, \mathrm{pH} 7.2, T_{0.5}=0.95 \mathrm{~s} ; \boldsymbol{\Delta}, \mathrm{pH} 6.9, T_{0.5}=2.61 \mathrm{~s} ; \square, \mathrm{pH} 6.7, T_{0.5}=6.06 \mathrm{~s}$. Inset, Enlargement on the first 10 s. D, Original type 2 current and potential traces recorded from one single dorsal spinal neuron. Depolarizations were activated by pH drops to pH 6.0 from pH 7.4 (top), pH 7.2 (middle), and pH 6.9 (bottom). For each resting $\mathrm{pH}$ value, several traces are superimposed, showing one control depolarization and several subsequent reactivated depolarizations recorded after different time intervals (s) from the end of the control pH drop: $0.1,0.3$, and $2 \mathrm{~s}$ at pH 7.4; $0.4,0.7,1,2$, and $4 \mathrm{~s}$ at pH 7.2 ; and 1, 2, 3, 5, and $10 \mathrm{~s}$ at pH 6.9. Only the first pH drop corresponding to the control depolarization is figured by a black line. The $0 \mathrm{mV}$ level is figured by a dashed line. Inset, Superimposed currents traces recorded at $-50 \mathrm{mV}$ and activated by pH drops from pH 7.4 to $\mathrm{pH} 6.0$ after different time intervals (1, $2,3 \mathrm{~s}$ ) from the end of the control (first) $\mathrm{pH}$ drop.

$\mathrm{pH}$-dependent regulation of the Type 2 current reactivation has dramatic effects on neuronal depolarization measured in the current-clamp mode (Fig. 4C,D). An extracellular $\mathrm{pH}$ decrease from 7.4 to 6.7 induced a 36-fold slowing-down of the depolarization reactivation with $T_{0.5}$ values increasing from 0.17 up to $6.1 \mathrm{~s}$. When $\mathrm{pH}$ was increased from $\mathrm{pH} 7.4$ to $\mathrm{pH} 9.0$, it induced a 5.7-fold acceleration of reactivation, with $T_{0.5}$ decreasing from 0.17 to $0.03 \mathrm{~s}$. Such a rapid reactivation, at the limit of our experimental resolution, corresponds to a near suppression of the neuronal refractory period in response to a $\mathrm{pH}$ drop. The different time scales between Figure $4 A$ and $C$ result from the classical nonlinear relationship between current amplitude and the subsequent induced depolarization (only a fraction of the maximal current induces the maximal depolarization). Original membrane potential recordings (Fig. 4D) illustrate the consequences of a light extracellular acidosis ( $\mathrm{pH}$ decreasing from 7.4 to 6.9) on the reactivation of type 2 current-induced depolarization and subsequent action potential triggering. A spinal neuron that is capable of coding for a $3 \mathrm{~Hz}$ acid stimulation ( $0.3 \mathrm{~s}$ time interval between two APs) from the extracellular $\mathrm{pH} 7.4$ to $\mathrm{pH} 6.0$ (Fig. $4 D$, top recordings) becomes only able to code for a $0.3 \mathrm{~Hz}$ acid stimulation ( $3 \mathrm{~s}$ time interval between two APs) from the extracellular pH 6.9 to $\mathrm{pH} 6.0$ (Fig. $4 \mathrm{D}$, bottom recording).

Similar results were observed on pure homomeric ASIC1a current recorded in ASIC2 ${ }^{-1-}$ neurons (Fig. 5), even if the reactivation $T_{0.5}$ values were globally larger than those of ASIC1a plus 

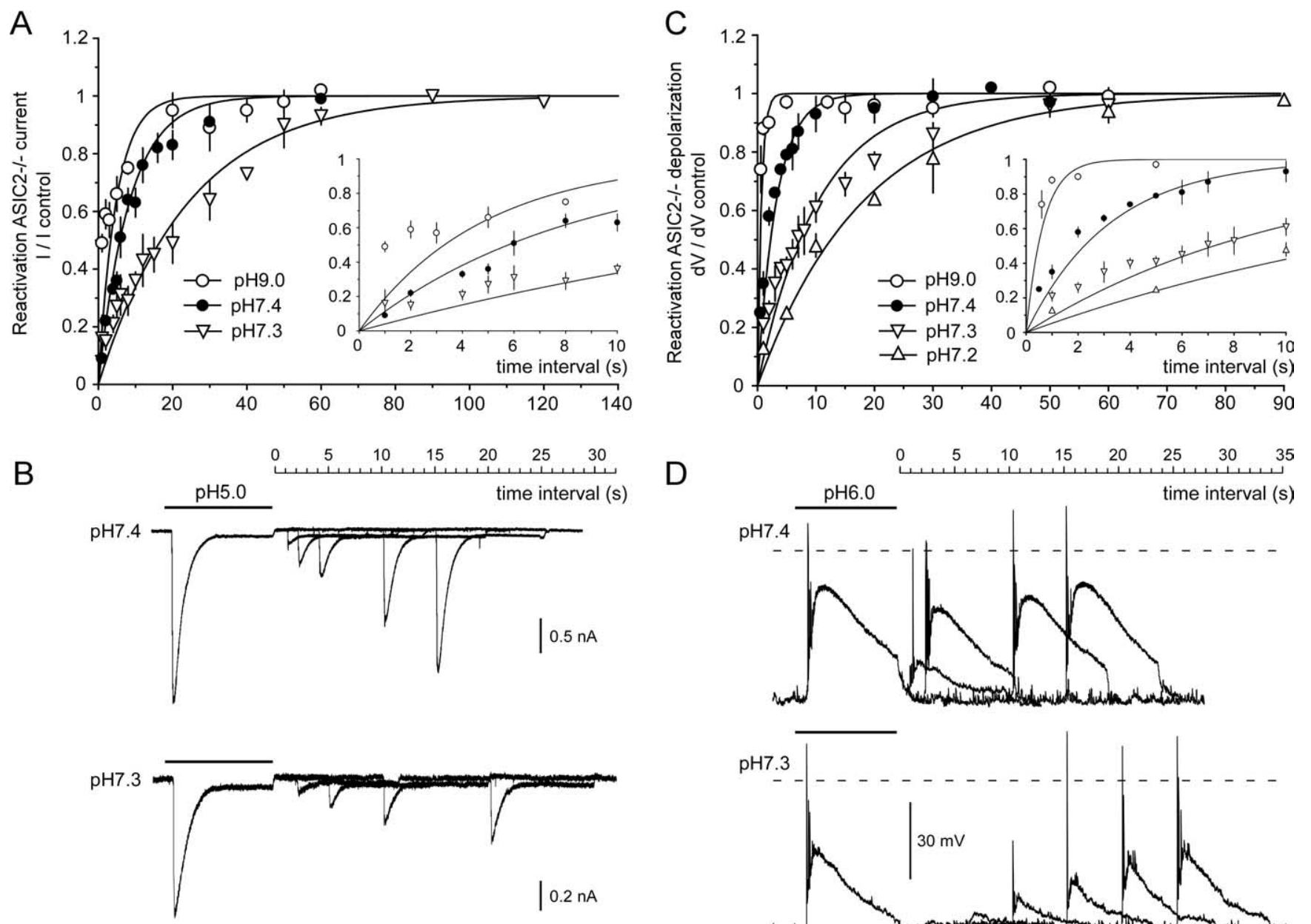

D
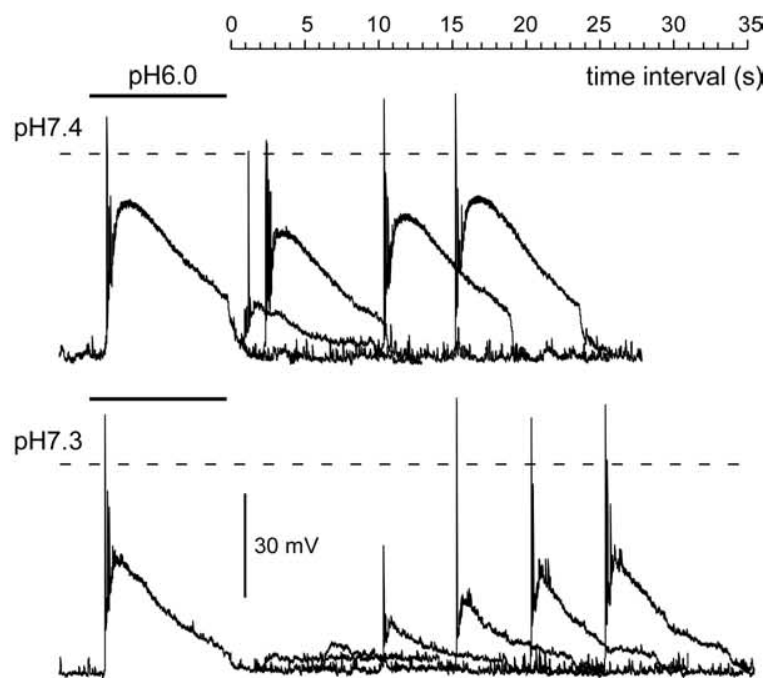

Figure 5. Modulation of the reactivation time course of dorsal spinal neuron $\mathrm{ASIC}^{-1-}$ current by extracellular pH and consequences on $\mathrm{AP}$ triggering. $\mathrm{A}$, $\mathrm{pH}-\mathrm{dependent} \mathrm{reactivation} \mathrm{of} \mathrm{ASIC2}{ }^{-1-}$ current. Currents were recorded at a holding potential of $-50 \mathrm{mV}$ and activated by $\mathrm{pH}$ drops from various resting $\mathrm{pH}$ values to $\mathrm{pH}$ 5.0. The current amplitude was expressed as a ratio of the amplitude of the control current (I// control) and plotted as mean \pm SEM ( $n$ ranging from 3 to 20 ) as a function of the time interval (s) between the end of the control pH drop and the onset of the next pH drop. Data were fitted as a one-phase exponential following the equation: $Y=Y \max ^{*}\left[1-\exp \left(-k^{*} t\right)\right]$, with half reactivation time $T_{0.5}=0.69 / k . \bigcirc, \mathrm{pH} 9.0, T_{0.5}=3.3 \mathrm{~s} ; \boldsymbol{\bullet}, \mathrm{pH} 7.4, T_{0.5}=5.77 \mathrm{~s} ; \nabla$, $\mathrm{pH} 7.3, T_{0.5}=16.84 \mathrm{~s}$. Inset, Enlargement on the first $10 \mathrm{~s}$. B, Original current traces recorded from one single ASIC2 ${ }^{-1-}$ neuron. Currents were recorded at $-50 \mathrm{mV}$ and activated by pH drops to pH 5.0 from pH 7.4 (top) and pH 7.3 (bottom). For each resting pH value, several traces are superimposed, showing one control current and several subsequent reactivated currents recorded after different time intervals (s) from the end of the control pH drop: 1, 2, 4, 10, and $15 \mathrm{~s}$ for pH 7.4; and 2, 5, 10, and $20 \mathrm{~s}$ for pH 7.3. Only the first pH drop corresponding to the control current is figured by a black line. $\mathrm{C}$, $\mathrm{pH}$-dependent reactivation of $\mathrm{ASIC}{ }^{-1-}$ current-induced depolarization. Depolarizations were recorded in current-clamp mode and activated by $\mathrm{pH}$ drops from various resting $\mathrm{pH}$ values to $\mathrm{pH}$ 6.0. The depolarization amplitude was expressed as a ratio of the control depolarization ( $d V / d V$ control) and plotted as mean \pm SEM ( $n$ ranging from 3 to 18 ) as a function of the time interval (s) between the end of the control pH drop and the onset of the next pH drop. Data were fitted as a one-phase exponential following the equation: $Y=Y \max ^{*}\left[1-\exp \left(-k^{*} t\right)\right]$, with half reactivation time $T_{0.5}=0.69 / \mathrm{k} . \bigcirc, \mathrm{pH} 9.0, T_{0.5}=0.46 \mathrm{~s} ; \boldsymbol{Q}, \mathrm{pH} 7.4, T_{0.5}=2.24 \mathrm{~s} ; \nabla, \mathrm{pH} 7.3, T_{0.5}=7.19 \mathrm{~s} ; \triangle, \mathrm{pH} 7.2, T_{0.5}=12.55 \mathrm{~s}$. Inset, Enlargement on the first $10 \mathrm{~s}$. $D$, Original potential traces recorded from one single dorsal spinal ASIC2 ${ }^{-1-}$ neuron. Depolarizations were activated by pH drops to $\mathrm{pH} 6.0$ from pH 7.4 (top) and pH 7.3 (bottom). For each resting pH value, several traces are superimposed, showing one control depolarization and several subsequent reactivated depolarizations recorded after different time intervals $(\mathrm{s})$ from the end of the control $\mathrm{pH}$ drop: $0.5,2,10$, and $15 \mathrm{~s}$ at pH 7.4; and 2,6,10,15,20, and $25 \mathrm{~s}$ at pH 7.3. Only the first pH drop corresponding to the control depolarization is figured by a black line. The $0 \mathrm{mV}$ level is figured by a dashed line.

2a heteromeric channels carrying type 2 current, and if the active range of homomeric ASICla channels was limited by their $\mathrm{pH}$ dependent inactivation process $\left(\mathrm{pH}_{0.5}=7.3\right)$. A very light acidosis from $\mathrm{pH} 7.4$ to $\mathrm{pH} 7.3$ increased $T_{0.5}$ from 5.77 up to $16.84 \mathrm{~s}$, whereas an increase in extracellular $\mathrm{pH}$ to $\mathrm{pH} 9.0$ reduced $T_{0.5}$ to $3.3 \mathrm{~s}$ (Fig. 5A). Representative original current traces are shown on Figure $5 B$. The $\mathrm{pH}$-dependent reactivation of homomeric ASIC1a channels also regulates neuronal excitability (Fig. 5C,D). An extracellular $\mathrm{pH}$ decrease from 7.4 to 7.3 induced a threefold slowing down of the depolarization reactivation with $T_{0.5}$ values increasing from 2.24 up to $7.19 \mathrm{~s}$, and a $\mathrm{pH}$ decrease from 7.4 to 7.2 induced a sixfold slowing down with $T_{0.5}$ reaching $12.55 \mathrm{~s}$. When $\mathrm{pH}$ was increased from $\mathrm{pH} 7.4$ to $\mathrm{pH} 9.0$, it induced a 4.9-fold acceleration of reactivation with $T_{0.5}$ decreasing from 2.24 to $0.46 \mathrm{~s}$. As a result of this channel behavior, a spinal neuron that is capable of coding for a $2 \mathrm{~Hz}$ acid stimulation $(0.5 \mathrm{~s}$ time interval between two APs) from the extracellular $\mathrm{pH} 7.4$ to $\mathrm{pH} 6.0$ (Fig. $5 D$, top traces) becomes only able to code for a $0.1 \mathrm{~Hz}$ acid stimulation (10 s time interval between two APs) from the extracellular pH 7.3 to pH 6.0 (Fig. 5D, bottom traces). A difference of only $0.1 \mathrm{pH}$ unit in the resting $\mathrm{pH}$ thus appears to induce drastic consequences on the firing properties after transient acidification to the same $\mathrm{pH}$ value.

These results were confirmed by experiments on heterologously expressed channels (supplemental Fig. 1, available at www.jneurosci.org as supplemental material). Homomeric ASIC1a channel reactivation is regulated by extracellular $\mathrm{pH}$, with $T_{0.5}$ increasing from 11.05 to $23.43 \mathrm{~s}$, when $\mathrm{pH}$ decreases from 7.4 to 7.2 , and $T_{0.5}$ decreasing to $3.01 \mathrm{~s}$ when $\mathrm{pH}$ increases up to 9.0 (supplemental Fig. $1 A$, available at www.jneurosci.org 


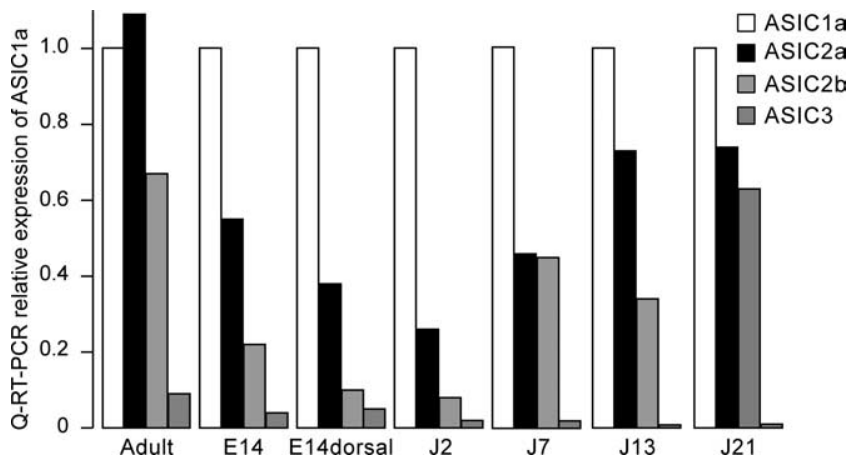

Figure 6. Relative expression of ASIC mRNAs in mouse spinal cord. Quantitative RT-PCR realized on total RNA extracts from adult spinal cord (Adult), fetal E14 spinal cord (E14), fetalE14 dorsal spinal cord (E14 dorsal), and primary cultured neurons from fetal E14 dorsal spinal cord after 2 (J2), 7 (J7), 13 (J13), and 21 (J21) days of culture. Expression of each ASIC is expressed as a ratio of the expression of ASIC1a in the same extract.

as supplemental material). Homomeric ASIC2a current reactivation also shows a marked $\mathrm{pH}$ dependency (supplemental Fig. $1 B$, available at www.jneurosci.org as supplemental material), with an 11 -fold increase in $T_{0.5}$ from 0.92 to $10.46 \mathrm{~s}$ when $\mathrm{pH}$ decreases from 7.4 to 6.6. A $T_{0.5}$ value of $153 \mathrm{~s}$ could be estimated at $\mathrm{pH}$ 6.0. When extracellular $\mathrm{pH}$ was increased from $\mathrm{pH} 7.4$ to $\mathrm{pH} 9.0$, it induced a marked acceleration of reactivation, $T_{0.5}$ decreasing from 0.92 to $0.03 \mathrm{~s}$. The $T_{0.5}$ values obtained at $\mathrm{pH} 7.4$ for ASIC1a and ASIC2a are in good agreement with previously published data (Benson et al., 2002). Coexpressing ASIC1a and ASIC2a with a 2:1 transfection ratio leads to a current with a $\mathrm{pH}$-dependent reactivation close to the neuronal type 2 current, a decrease in extracellular $\mathrm{pH}$ from 7.4 to 6.6 inducing a 36 -fold increase in the $T_{0.5}$ of the ASIC1a plus 2a current from 0.5 to $18.08 \mathrm{~s}$ (supplemental Fig. $1 C$, available at www.jneurosci.org as supplemental material). These results further support the participation of heteromeric ASIC1a plus 2a channels in the peak type 2 current in dorsal spinal neurons, and the involvement of both subunits in their $\mathrm{pH}$-dependent reactivation.

\section{ASIC1a and ASIC2a are the most abundant ASICs expressed in spinal neurons where they are coexpressed in most neuronal subtypes}

Our functional data suggest a predominant role for the ASIC1a and ASIC2a subunits in mouse spinal cord. These isoforms have already been detected in the spinal cord (Chen et al., 1998; Alvarez de la Rosa et al., 2003; Wu et al., 2004), but their pattern of expression and their relative abundance remained to be determined. We compared by quantitative RT-PCR the expression of the ASIC isoforms between adult spinal cord, E14 spinal cord, dorsal E14 spinal cord, and primary cultured dorsal E14 spinal neurons used for patch-clamp experiments (Fig. 6). As described previously (Wu et al., 2004), ASIC1a, ASIC2a, and to a lower extent ASIC2b were the major mRNAs detected in embryonic and adult spinal cord, whereas ASIC3 expression was negligible, as well as ASIC1b and TRPV1 expression (data not shown), consistent with their specific expression in sensory neurons. In good agreement with these data, we failed to detect ASIC3-like currents in spinal cord neurons (data not shown). ASICla appears as the most abundant isoform detected in E14 spinal cord, ASIC2a and ASIC2b representing, respectively, 55 and $24 \%$ of ASIC1a. ASIC1a is also the most abundant isoform in the dorsal half of the E14 spinal cord, with ASIC2a and ASIC2b representing 38 and $10 \%$ of ASIC1a, respectively. This pattern did not change after $2 \mathrm{~d}$ of culture, but with time, it progressively evolved toward a pattern close to the one found in the adult spinal cord, with an increase in ASIC2a and ASIC2b proportions (ASIC2a and ASIC2b representing 74 and $63 \%$ of ASIC1a after $21 \mathrm{~d}$ of culture). In addition to a change in the respective expression pattern of ASIC1a, ASIC2a, and ASIC2b with time in culture, the level of expression of all ASICs also progressively increased. ASIC1a expression was increased 2.5-fold, 4.7-fold, and 5.3-fold compared with E14 dorsal spinal cord after 7, 13, and $21 \mathrm{~d}$ in culture (data not shown).

We next performed in situ hybridization experiments to determine the spatial pattern of expression of ASIC1a and ASIC2a in adult spinal cord and showed that ASICla (Fig. 7A,a) and ASIC2a (Fig. $7 A, b$ ) are expressed by neurons throughout all spinal laminas (Fig. $7 A, c)$; by the small $(\sim 10 \mu \mathrm{m}$ in diameter) and closely spaced neurons of dorsal laminas 1-3 (substantia gelatinosa) receiving the peripheral nociceptive afferent fibers; by the medium size ( $\sim 20 \mu \mathrm{m}$ in diameter) wide-dynamic range (WDR) neurons of dorsal laminas $4-5$, which are the main origin of the spinothalamic tract conveying sensory information to brain; and by ventral big size $(30-40 \mu \mathrm{m}$ in diameter) motoneurons of laminas 8-9. Control experiments with the sense probe are shown in supplemental Figure 2 (available at www.jneurosci.org as supplemental material). Labeling of ASIC1a by fluorescent in situ hybridization and codetection of ASIC2a by immunohistochemistry (Fig. $7 B$ ) showed an extensive colocalization of ASIC1a mRNA and ASIC2a protein in spinal cord neurons, consistent with electrophysiological data showing an important participation of ASIC1a plus 2a heteromers in spinal cord ASIC currents.

\section{Discussion}

ASIC1a has very recently emerged as a key subunit in the modulation of nociception in the CNS (Duan et al., 2007; Mazzuca et al., 2007). Our data confirm the importance of the ASICla subunit in spinal cord neurons. ASIC1a is broadly expressed in all the laminas of the adult spinal cord and contributes to every ASIC currents recorded from cultured spinal cord neurons. It was very recently suggested by Duan et al. (2007) that homomeric ASIC1a is the major channel underlying ASIC currents in rat spinal dorsal horn neurons, with no functional participation of ASIC2a. We show here that ASIC1a homomers are actually not the predominant functional channels in these neurons, in good agreement with the very significant expression of ASIC2a and ASIC2b in spinal cord (Wu et al., 2004; our study). ASIC2a is broadly expressed in all the laminas of the adult spinal cord, where it is often colocalized with ASIC1a, and 83\% of E14 cultured mouse dorsal spinal neurons display transient currents resembling heteromeric ASIC1a plus $2 \mathrm{a}$ channel (i.e., type 2 and type 3 currents). This heteromeric channel is most probably formed of two ASICla and one ASIC2a subunits, based on our comparison between the properties of recombinant channels expressed at different ratio and native currents in dorsal spinal neurons, as well as on the recently described trimeric organization of ASIC channels obtained from the determination of the tridimensional structure of ASIC1 (Jasti et al., 2007).

We cannot completely exclude a modulatory role for ASIC2b in spinal cord. The formation of heterotrimers comprising ASIC2b together with ASIC1a and/or ASIC2a is conceivable given the lack of data on the modulation of ASIC2b-containing channels by zinc and the contribution of ASIC2b to the plateau phase sometimes associated with type 2 current. However, ASIC2b seems to play a minor role in this current considering the biophysical properties of type 2 current and the suggested com- 
position of the major underlying channel with two ASIC1a subunits and one ASIC2a subunit.

The role of ASICs in the spinal cord is probably complex, because these channels could be involved at different levels in pain processing. ASICs can modulate the transmission of the noxious message either directly or indirectly. Our group has shown recently that ASICla homomeric channels in the CNS are involved in the control of the endogenous opioid system by a still unknown mechanism (Mazzuca et al., 2007). Blockade of ASICla by intrathecal injection of the toxin PcTx1 or by antisense oligonucleotides results in activation of the endogenous enkephalin pathway and is associated with a potent analgesic effect (Mazzuca et al., 2007). Duan et al. (2007) also have described recently that intrathecal injection of the venom of the tarantula Psalmopoeus cambridgei that contains PcTx1 (among many other components), or of antisense oligonucleotides, reverse inflammatory pain hypersensitivity in rat. Because PcTxl specifically blocks ASICla homomeric channels (Escoubas et al., 2000), it is probable that the effect of intrathecally injected PcTxl involves the dorsal spinal neurons displaying type 1 and/or type 3 currents. The presence of ASIC2a in ASIC channels prevents their inhibition by PcTx1 (Escoubas et al., 2000). The type 2 current, which is predominant in $\sim 66 \%$ of cultured spinal cord neurons, is thus insensitive to PcTx1 and therefore does not participate to the potent pharmacological in vivo effects of the toxin. This suggests that ASIC2a and type 2 currents might not be major contributors to nociceptive processing in the spinal cord. However, a definitive answer will be obtained only when a specific blocker of type 2 current will be available. Blocking the type 1 current with PcTxl is sufficient to have a potent analgesic effect through strong endogenous opioid-mediated mechanisms, but this does not mean that a specific block of type 2 current would not also induce analgesia.

Both ASIC1a and ASIC2a are present at the synapse (Duggan et al., 2002; Wemmie et al., 2002; Jovov et al., 2003). ASIC1acontaining homomeric or heteromeric channels are thought to be activated by the release of the acidic content of synaptic vesicles during neuronal activity and the subsequent acidification in the vicinity of the synaptic cleft (Krishtal et al., 1987; Chesler and Kaila, 1992; Waldmann et al., 1997; Waldmann and Lazdunski, 1998; Krishtal, 2003), but no direct demonstration of a participation of these channels in synaptic transmission has been provided thus far. ASIC1a properties are drastically modified by its association with ASIC2a, even in minor proportion (1:2 ratio) within heteromers. In addition to conferring a coactivation by $\mathrm{H}^{+}$and $\mathrm{Zn}^{2+}$ (Baron
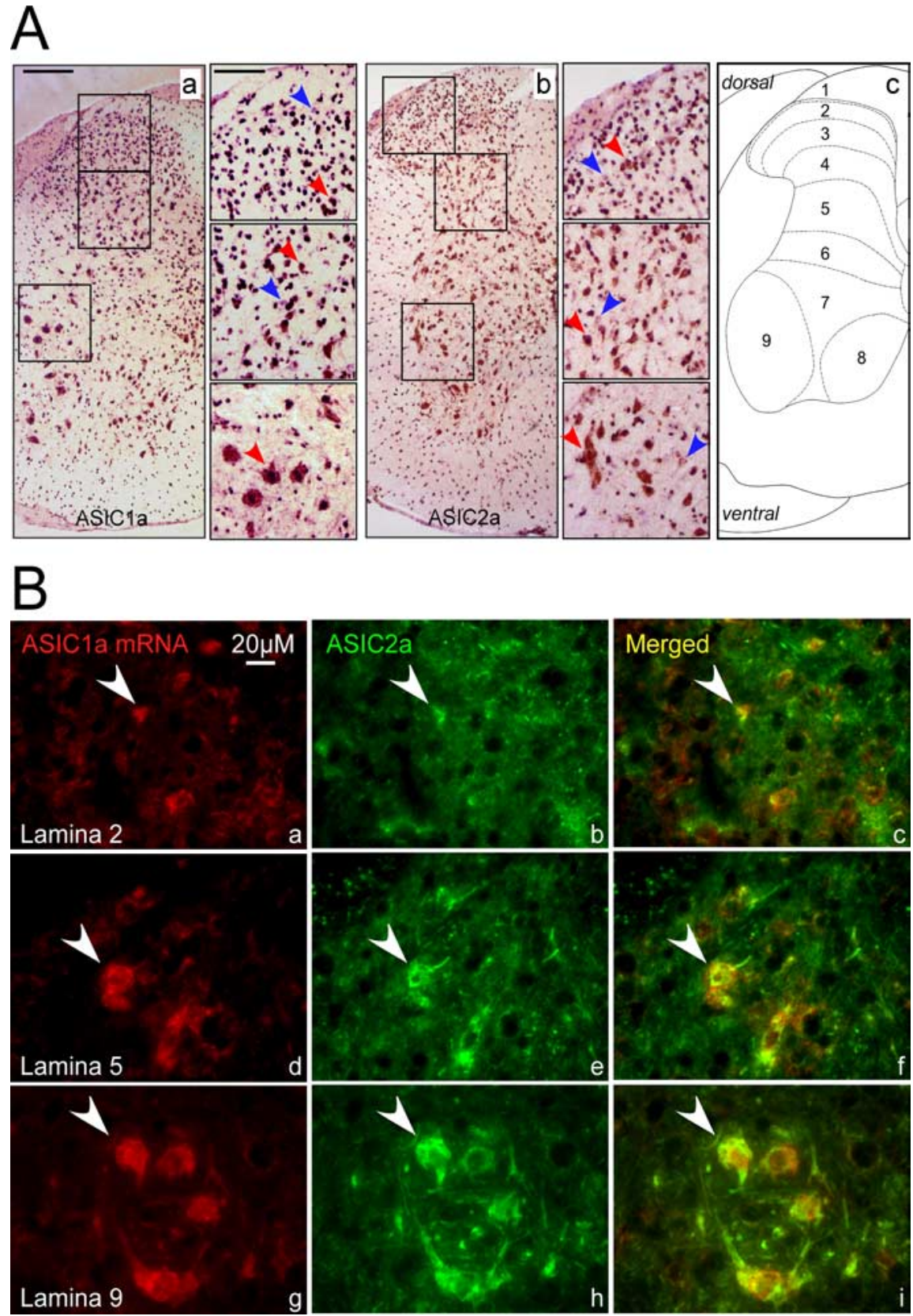

Figure 7. Localization of ASIC1a and ASIC2a in mouse adult spinal cord. $\boldsymbol{A}$, In situ hybridization of ASIC1a (a) and ASIC2a (b) on adult spinal cord sections, detected using DAB with a light cresyl violet counter-staining. For each ASIC, a hemi-spinal cord is shown (scale bar, $200 \mu \mathrm{m}$ ) along with three enlargements (scale bar, $100 \mu \mathrm{m}$ ) localized by black boxes on the corresponding hemi-spinal cord. DAB-labeled cells appear in brown to black (red arrow), and DAB-unlabeled cells appear in pink to violet (blue arrow). Orientation of the slices and position of one to nine laminas are figured on a drawing (c) of the spinal cord at the lumbar 5 level. $\boldsymbol{B}$, Immunohistolabeling of ASIC 2 a (green) was performed on the same adult spinal cord section than in situ hybridization for ASIC1a detected by immunofluorescence (red). Labeling for ASIC1a $(\boldsymbol{a}, \boldsymbol{d}, \boldsymbol{g}), \operatorname{ASIC2a}(\boldsymbol{b}, \boldsymbol{e}, \boldsymbol{h})$, and the merged image $(\boldsymbol{c}, \boldsymbol{f}, \boldsymbol{i})$ is shown in lamina $2(\boldsymbol{a}-\boldsymbol{c})$, lamina $5(\boldsymbol{d}-\boldsymbol{f})$, and lamina $9(\boldsymbol{g}-\boldsymbol{i})$ of the same spinal cord. White arrows indicate examples of double-labeled neurons. Scale bar, $20 \mu \mathrm{m}$.

et al., 2001), the ASIC2a subunit determines the active $\mathrm{pH}$ and kinetics of heteromeric ASIC1a plus 2a channels. ASIC2a can also bring into the ASICla plus 2 a complex other associated proteins capable of modulating the channel activity, such as protein kinase $\mathrm{C}$ (PKC)-interacting protein 1 (PICK1) that confers a regulation by PKC $\alpha$ (Baron et al., 2002b). As in the hippocampus, glutamatergic synapses in the dorsal spinal cord corelease protons that could activate ASIC channels, as well as $\mathrm{Zn}^{2+}$, released both from afferent sensory small-diameter neurons and from some dorsal 
horn neurons particularly in superficial laminas (Velazquez et al., 1999; Jo et al., 2000; Danscher et al., 2001; Perez-Castejon et al., 2002). Interestingly, $\mathrm{Zn}^{2+}$, PICK1, and PKC have been involved in C fiber-evoked LTP (long-lasting increase in synaptic strength) in the dorsal horn (Schroder et al., 2000; Ma and Zhao, 2001) as well as in dorsal spinal WDR neurons (Martin et al., 1999; Woolf and Salter, 2000; Vikman et al., 2001; Mantyh et al., 2002; Ji et al., 2003; Rygh et al., 2005). PKC $\alpha$ shows a broad distribution in the dorsal spinal cord, particularly in laminas 1-3 (Akinori, 1998), where it is expressed at synapses (Mori et al., 1990), and PICK1 may thus act to bring PKC $\alpha$ close to plasma membrane substrates such as ASIC channels. Interestingly, PICK1 has been involved in neuropathic sensitization in rats (Garry et al., 2003), whereas vesicular $\mathrm{Zn}^{2+}$ in dorsal horn has been associated recently with neuropathic pain in mice (Jo et al., 2007). Synaptic plasticity processes are relevant to central sensitization in the spinal cord (Julius and Basbaum, 2001; Wu et al., 2004). There is considerable similarity with hippocampal LTP (Sandkuhler, 2007), which is impaired in ASIC1inactivated mice (Wemmie et al., 2002), but the involvement of spinal ASIC channels in A $\delta$ - and/or C fiber-induced LTP remains to be demonstrated. Homomeric ASICla channels have been involved recently in C fiber-induced wind-up (transient increase in spike frequency) in WDR dorsal spinal neurons (Duan et al., 2007), another phenomenon contributing to central sensitization.

Analysis of the properties of spinal cord neuron ASIC currents revealed an important and new property of these channels shared by homomeric ASIC1a and ASIC2a as well as by heteromeric ASIC1a plus 2a channels (i.e., the regulation of ASIC channel reactivation kinetics by extracellular $\mathrm{pH}$ ). In spinal dorsal neurons, the two types of ASIC currents (type 1 and type 2) show dramatically different time courses of reactivation at the resting $\mathrm{pH}$ 7.4. Homomeric ASIC1a current (recorded in ASIC2 ${ }^{-1-}$ neurons) is very slow to reactivate $\left(T_{0.5}=5.5 \mathrm{~s}\right.$; full reactivation in $35 \mathrm{~s}$ ), whereas ASIC1a plus 2a heteromers, carrying the most abundant current in spinal cord (i.e., type 2 current), reactivate with a $T_{0.5}=0.98 \mathrm{~s}$. Thus, the type 2 current has functional properties more compatible with high-frequency stimulations. In the CNS, short high-frequency action potential bursts are predominant information carriers, believed to be associated with information processing and memory consolidation (Krishtal, 2003), and, as indicated previously, hippocampal synaptic facilitation by high-frequency burst is altered in the $\mathrm{ASICla}^{-1-}$ mice (Wemmie et al., 2002). C fibers usually discharge at $\sim 1-10 \mathrm{~Hz}$, and such stimulations induce LTP at C-fiber synapses in spinal neurons from laminas 1-5 (Ikeda et al., 2006; Sandkuhler, 2007), which express both ASIC1a and ASIC2a as shown in this paper. An increase in excitatory synaptic inputs of dorsal spinal neurons, particularly in lamina 2, is also frequently observed in various pathological states, such as peripheral inflammation (Furue et al., 2004), which is associated with an increased ASIC expression in dorsal spinal neurons (Wu et al., 2004; Duan et al., 2007). Acidosis slows down, whereas alkalinization accelerates reactivation of ASIC currents in the spinal cord. The steep pH-dependent regulation is expected to play an important modulatory role for neuronal excitability in relationship with the ability of ASIC currents to trigger action potentials, and thus repetitive firing in response to fast $\mathrm{pH}$ oscillations induced by an intense synaptic activity and/or pathological states. As mentioned previously, ASIC1a homomers contribute to the wind-up induced by a $0.5 \mathrm{~Hz}$ stimulation of C-fibers in WDR spinal neurons (Duan et al., 2007). Considering the different functional properties of ASICla homomers and ASIC1a plus 2a heteromers, it is tempting to propose that ASIC1a homomers code for low-frequency synaptic inputs not associated with a sustained acidification that inactivates the channels, whereas ASIC1a plus 2a heteromers code for both low- and high-frequency inputs at resting $\mathrm{pH} 7.4$ but remain able to code for low-frequency spikes when a sustained extracellular acidification takes place, for instance during intense synaptic activity (Fig. $4 D$ ). By slowing down the reactivation of ASIC current, a sustained extracellular acidification would decrease their ability to induce repetitive firing in neurons of the spinal cord, thus constituting a protective mechanism during intense pain transmission associated with pathological states or tissue damage. At this stage, it is probably important to point out that ASIC currents in hippocampus neurons also predominantly flow through ASIC1a plus 2 a heteromers (Baron et al., 2002a). Therefore, the modulation of ASIC reactivation time course by extracellular $\mathrm{pH}$ probably provides an important new mechanism to finely modulate neuronal excitability not only in the spinal cord but also in the brain, particularly in pathological situations such as chronic pain, but also ischemia or seizures.

\section{References}

Akinori M (1998) Subspecies of protein kinase C in the rat spinal cord. Prog Neurobiol 54:499-530

Akopian AN, Chen CC, Ding Y, Cesare P, Wood JN (2000) A new member of the acid-sensing ion channel family. NeuroReport 11:2217-2222.

Alvarez de la Rosa D, Krueger SR, Kolar A, Shao D, Fitzsimonds RM, Canessa CM (2003) Distribution, subcellular localization and ontogeny of ASIC1 in the mammalian central nervous system. J Physiol (Lond) 546:77-87.

Askwith CC, Wemmie JA, Price MP, Rokhlina T, Welsh MJ (2004) Acidsensing ion channel 2 (ASIC2) modulates ASIC1 $\mathrm{H}^{+}$-activated currents in hippocampal neurons. J Biol Chem 279:18296-18305.

Baron A, Schaefer L, Lingueglia E, Champigny G, Lazdunski M (2001) $\mathrm{Zn}^{2+}$ and $\mathrm{H}^{+}$are coactivators of acid-sensing ion channels. J Biol Chem 276:35361-35367.

Baron A, Waldmann R, Lazdunski M (2002a) ASIC-like, proton-activated currents in rat hippocampal neurons. J Physiol (Lond) 539:485-494.

Baron A, Deval E, Salinas M, Lingueglia E, Voilley N, Lazdunski M (2002b) Protein kinase $\mathrm{C}$ stimulates the acid-sensing ion channel ASIC2a via the PDZ domain-containing protein PICK1. J Biol Chem 277:50463-50468.

Benson CJ, Xie J, Wemmie JA, Price MP, Henss JM, Welsh MJ, Snyder PM (2002) Heteromultimers of DEG/ENaC subunits form $\mathrm{H}^{+}$-gated channels in mouse sensory neurons. Proc Natl Acad Sci USA 99:2338-2343.

Chen CC, England S, Akopian AN, Wood JN (1998) A sensory neuronspecific, proton-gated ion channel. Proc Natl Acad Sci USA 95:10240-10245.

Chesler M, Kaila K (1992) Modulation of pH by neuronal activity. Trends Neurosci 15:396-402.

Chu XP, Wemmie JA, Wang WZ, Zhu XM, Saugstad JA, Price MP, Simon RP, Xiong ZG (2004) Subunit-dependent high-affinity zinc inhibition of acid-sensing ion channels. J Neurosci 24:8678-8689.

Danscher G, Jo SM, Varea E, Wang Z, Cole TB, Schroder HD (2001) Inhibitory zinc-enriched terminals in mouse spinal cord. Neuroscience 105:941-947.

Duan B, Wu LJ, Yu YQ, Ding Y, Jing L, Xu L, Chen J, Xu TL (2007) Upregulation of acid-sensing ion channel ASICla in spinal dorsal horn neurons contributes to inflammatory pain hypersensitivity. J Neurosci 27:11139-11148.

Duggan A, Garcia-Anoveros J, Corey DP (2002) The PDZ domain protein PICK1 and the sodium channel $\mathrm{BNaC} 1$ interact and localize at mechanosensory terminals of dorsal root ganglion neurons and dendrites of central neurons. J Biol Chem 277:5203-5208.

Duprat F, Lesage F, Fink M, Reyes R, Heurteaux C, Lazdunski M (1997) TASK, a human background $\mathrm{K}^{+}$channel to sense external $\mathrm{pH}$ variations near physiological pH. EMBO J 16:5464-5471.

Escoubas P, De Weille JR, Lecoq A, Diochot S, Waldmann R, Champigny G, Moinier D, Menez A, Lazdunski M (2000) Isolation of a tarantula toxin specific for a class of proton-gated $\mathrm{Na}^{+}$channels. J Biol Chem 275:25116-25121.

Escoubas P, Bernard C, Lambeau G, Lazdunski M, Darbon H (2003) Recombinant production and solution structure of PcTxl, the specific pep- 
tide inhibitor of ASICla proton-gated cation channels. Protein Sci 12:1332-1343.

Ettaiche M, Guy N, Hofman P, Lazdunski M, Waldmann R (2004) Acidsensing ion channel 2 is important for retinal function and protects against light-induced retinal degeneration. J Neurosci 24:1005-1012.

Ettaiche M, Deval E, Cougnon M, Lazdunski M, Voilley N (2006) Silencing acid-sensing ion channel la alters cone-mediated retinal function. J Neurosci 26:5800-5809.

Furue H, Katafuchi T, Yoshimura M (2004) Sensory processing and functional reorganization of sensory transmission under pathological conditions in the spinal dorsal horn. Neurosci Res 48:361-368.

Garcia-Anoveros J, Derfler B, Neville-Golden J, Hyman BT, Corey DP (1997) $\mathrm{BNaC} 1$ and $\mathrm{BNaC} 2$ constitute a new family of human neuronal sodium channels related to degenerins and epithelial sodium channels. Proc Natl Acad Sci USA 94:1459-1464.

Garry EM, Moss A, Rosie R, Delaney A, Mitchell R, Fleetwood-Walker SM (2003) Specific involvement in neuropathic pain of AMPA receptors and adapter proteins for the GluR2 subunit. Mol Cell Neurosci 24:10-22.

Grunder S, Geissler HS, Bassler EL, Ruppersberg JP (2000) A new member of acid-sensing ion channels from pituitary gland. NeuroReport 11:1607-1611.

Gruol DL, Barker JL, Huang LY, MacDonald JF, Smith Jr TG (1980) Hydrogen ions have multiple effects on the excitability of cultured mammalian neurons. Brain Res 183:247-252.

Hamill OP, Marty A, Neher E, Sakmann B, Sigworth FJ (1981) Improved patch-clamp techniques for high-resolution current recording from cells and cell-free membrane patches. Pflügers Arch 391:85-100.

Hesselager M, Timmermann DB, Ahring PK (2004) pH dependency and desensitization kinetics of heterologously expressed combinations of acid-sensing ion channel subunits. J Biol Chem 279:11006-11015.

Ikeda H, Stark J, Fischer H, Wagner M, Drdla R, Jager T, Sandkuhler J (2006) Synaptic amplifier of inflammatory pain in the spinal dorsal horn. Science 312:1659-1662

Jasti J, Furukawa H, Gonzales EB, Gouaux E (2007) Structure of acidsensing ion channel 1 at 1.9 A resolution and low $\mathrm{pH}$. Nature 449:316-323.

Ji RR, Kohno T, Moore KA, Woolf CJ (2003) Central sensitization and LTP: do pain and memory share similar mechanisms? Trends Neurosci 26:696-705.

Jo SM, Danscher G, Daa Schroder H, Won MH, Cole TB (2000) Zincenriched (ZEN) terminals in mouse spinal cord: immunohistochemistry and autometallography. Brain Res 870:163-169.

Jo SM, Danscher G, Schroder HD, Suh SW (2007) Depletion of vesicular zinc in dorsal horn of spinal cord causes increased neuropathic pain in mice. Biometals, in press.

Jones NG, Slater R, Cadiou H, McNaughton P, McMahon SB (2004) Acidinduced pain and its modulation in humans. J Neurosci 24:10974-10979.

Jovov B, Tousson A, McMahon LL, Benos DJ (2003) Immunolocalization of the acid-sensing ion channel 2a in the rat cerebellum. Histochem Cell Biol 119:437-446.

Julius D, Basbaum AI (2001) Molecular mechanisms of nociception. Nature 413:203-210.

Krishtal O (2003) The ASICs: signaling molecules? Modulators? Trends Neurosci 26:477-483.

Krishtal OA, Osipchuk YV, Shelest TN, Smirnoff SV (1987) Rapid extracellular $\mathrm{pH}$ transients related to synaptic transmission in rat hippocampal slices. Brain Res 436:352-356.

Li YX, Schaffner AE, Li HR, Nelson R, Barker JL (1997) Proton-induced cation current in embryonic rat spinal cord neurons changes ion dependency over time in vitro. Brain Res Dev Brain Res 102:261-266.

Lingueglia E (2007) Acid-sensing ion channels in sensory perception. J Biol Chem 282:17325-17329.

Lingueglia E, de Weille JR, Bassilana F, Heurteaux C, Sakai H, Waldmann R, Lazdunski M (1997) A modulatory subunit of acid sensing ion channels in brain and dorsal root ganglion cells. J Biol Chem 272:29778-29783.

Ma JY, Zhao ZQ (2001) The effects of $\mathrm{Zn}^{2+}$ on long-term potentiation of C fiber-evoked potentials in the rat spinal dorsal horn. Brain Res Bull $56: 575-579$.
Mantyh PW, Clohisy DR, Koltzenburg M, Hunt SP (2002) Molecular mechanisms of cancer pain. Nat Rev Cancer 2:201-209.

Martin WJ, Liu H, Wang H, Malmberg AB, Basbaum AI (1999) Inflammation-induced up-regulation of protein kinase $\mathrm{C}$ gamma immunoreactivity in rat spinal cord correlates with enhanced nociceptive processing. Neuroscience 88:1267-1274.

Mazzuca M, Heurteaux C, Alloui A, Diochot S, Baron A, Voilley N, Blondeau N, Escoubas P, Gelot A, Cupo A, Zimmer A, Zimmer AM, Eschalier A, Lazdunski M (2007) A tarantula peptide against pain via ASICla channels and opioid mechanisms. Nat Neurosci 10:943-945.

McCleskey EW, Gold MS (1999) Ion channels of nociception. Annu Rev Physiol 61:835-856.

Mori M, Kose A, Tsujino T, Tanaka C (1990) Immunocytochemical localization of protein kinase $\mathrm{C}$ subspecies in the rat spinal cord: light and electron microscopic study. J Comp Neurol 299:167-177.

Perez-Castejon MC, Vera-Gil A, Lahoz M, Aisa J, Recreo MP, Pes N, Serrano P, Barral MJ (2002) Location of zinc and 65Zn in spinal ganglia of the rat. Histol Histopathol 17:799-803.

Price MP, Snyder PM, Welsh MJ (1996) Cloning and expression of a novel human brain $\mathrm{Na}^{+}$channel. J Biol Chem 271:7879-7882.

Rygh LJ, Svendsen F, Fiska A, Haugan F, Hole K, Tjolsen A (2005) Longterm potentiation in spinal nociceptive systems-how acute pain may become chronic. Psychoneuroendocrinology 30:959-964.

Sandkuhler J (2007) Understanding LTP in pain pathways. Mol Pain 3:9.

Schroder HD, Danscher G, Jo SM, Su H (2000) Zinc-enriched boutons in rat spinal cord. Brain Res 868:119-122.

Varming T (1999) Proton-gated ion channels in cultured mouse cortical neurons. Neuropharmacology 38:1875-1881.

Velazquez RA, Cai Y, Shi Q, Larson AA (1999) The distribution of zinc selenite and expression of metallothionein-III mRNA in the spinal cord and dorsal root ganglia of the rat suggest a role for zinc in sensory transmission. J Neurosci 19:2288-2300.

Vikman KS, Kristensson K, Hill RH (2001) Sensitization of dorsal horn neurons in a two-compartment cell culture model: wind-up and long-term potentiation-like responses. J Neurosci 21:RC169(1-6).

Voilley N (2004) Acid-sensing ion channels (ASICs): new targets for the analgesic effects of non-steroid anti-inflammatory drugs (NSAIDs). Curr Drug Targets Inflamm Allergy 3:71-79.

Waldmann R, Lazdunski M (1998) H(+)-gated cation channels: neuronal acid sensors in the NaC/DEG family of ion channels. Curr Opin Neurobiol 8:418-424.

Waldmann R, Champigny G, Voilley N, Lauritzen I, Lazdunski M (1996) The mammalian degenerin MDEG, an amiloride-sensitive cation channel activated by mutations causing neurodegeneration in Caenorhabditis elegans. J Biol Chem 271:10433-10436.

Waldmann R, Champigny G, Bassilana F, Heurteaux C, Lazdunski M (1997) A proton-gated cation channel involved in acid-sensing. Nature 386:173-177.

Wemmie JA, Chen J, Askwith CC, Hruska-Hageman AM, Price MP, Nolan BC, Yoder PG, Lamani E, Hoshi T, Freeman Jr JH, Welsh MJ (2002) The acid-activated ion channel ASIC contributes to synaptic plasticity, learning, and memory. Neuron 34:463-477.

Wemmie JA, Askwith CC, Lamani E, Cassell MD, Freeman Jr JH, Welsh MJ (2003) Acid-sensing ion channel 1 is localized in brain regions with high synaptic density and contributes to fear conditioning. J Neurosci 23:5496-5502.

Wemmie JA, Price MP, Welsh MJ (2006) Acid-sensing ion channels: advances, questions and therapeutic opportunities. Trends Neurosci 29:578-586.

Woolf CJ, Salter MW (2000) Neuronal plasticity: increasing the gain in pain. Science 288:1765-1769.

Wu LJ, Duan B, Mei YD, Gao J, Chen JG, Zhuo M, Xu L, Wu M, Xu TL (2004) Characterization of acid-sensing ion channels in dorsal horn neurons of rat spinal cord. J Biol Chem 279:43716-43724.

Yang L, Zhang FX, Huang F, Lu YJ, Li GD, Bao L, Xiao HS, Zhang X (2004) Peripheral nerve injury induces trans-synaptic modification of channels receptors and signal pathways in rat dorsal spinal cord. Eur J Neurosci 19:871-883. 